\title{
Physical structure and dust reprocessing in a sample of $\mathrm{HH}$ jets $\star, \star \star$
}

\author{
L. Podio ${ }^{1}$, S. Medves ${ }^{2}$, F. Bacciotti ${ }^{3}$, J. Eislöffel ${ }^{4}$, and T. Ray ${ }^{1}$ \\ 1 Dublin Institute for Advanced Studies, School of Cosmic Physics, 31 Fitzwilliam Place, Dublin 2, Ireland \\ e-mail: lpodio@cp.dias.ie \\ 2 Università di Pisa, Dipartimento di Fisica "Enrico Fermi”, Largo Bruno Pontecorvo 3, 56127 Pisa, Italy \\ e-mail: silvia.medves@gmail.com \\ 3 INAF - Osservatorio Astrofisico di Arcetri, Largo Enrico Fermi 5, 50125 Firenze, Italy \\ e-mail: fran@arcetri.astro.it \\ 4 Thüringer Landessternwarte Tautenburg, Sternwarte 5, 07778 Tautenburg, Germany \\ e-mail: jochen@tls-tautenburg.de
}

Received 30 April 2009 / Accepted 21 July 2009

\begin{abstract}
Context. Stellar jets are an essential ingredient of the star formation process and a wealth of information can be derived from their characteristic emission-line spectra.

Aims. We investigate the physical structure and dust reprocessing in the shocks along the beam of a number of classical Herbig-Haro $(\mathrm{HH})$ jets in the Orion and Lupus molecular clouds (HH 111, HH 1/2, HH 83, HH 24 M/A/E/C, and Sz68). Parameters describing plasma conditions, as well as dust content, are derived as a function of distance from the source and, for HH 111, of gas velocity. Methods. Spectral diagnostic techniques are applied to obtain the jet physical conditions (the electron and total density, $n_{\mathrm{e}}$ and $n_{\mathrm{H}}$, the ionisation fraction, $x_{\mathrm{e}}$, and the temperature, $T_{\mathrm{e}}$ ) from the ratios of selected forbidden lines. The presence of dust grains is investigated by estimating the gas-phase abundance of calcium with respect to its solar value.

Results. We find the electron density varies between $0.05-4 \times 10^{3} \mathrm{~cm}^{-3}$, the ionisation fraction $x_{\mathrm{e}}$ from $0.01-0.7$, the temperature ranges between $0.6-3 \times 10^{4} \mathrm{~K}$, and the hydrogen density between $0.01-6 \times 10^{4} \mathrm{~cm}^{-3}$. Interestingly, in the $\mathrm{HH} 111$ jet, $n_{\mathrm{e}}, x_{\mathrm{e}}$, and $T_{\mathrm{e}}$ peak in the high velocity interval (HVI) of the strongest working surfaces, confirming a prediction from shocks models. Calcium turns out to be depleted with respect to its solar value, but its gas-phase abundance is higher than estimates for the interstellar medium in Orion. The depletion is high (up to 80\%) along the low-excited jets, while low or no depletion is measured in those jets which show higher excitation conditions. Moreover, for HH 111 the depletion is lower in the HVI of the fastest shock.

Conclusions. Our results confirm the shock structure predicted by models and indicate that shocks occurring along jets, and presumably those present in the launch zone, only partially destroy dust grains and that the efficiency of dust reprocessing strongly depends on shock velocity. However, the high Ca gas-phase abundance estimated in some of the knots, is not well understood in terms of existing models of dust reprocessing in shocks, and indicates that the dust must have been partially reprocessed in the region where the flow originates.
\end{abstract}

Key words. ISM: jets and outflows - ISM: Herbig-Haro objects - ISM: dust, extinction - stars: formation

\section{Introduction}

Although it is widely accepted that jets play an essential role in star formation, there are still many open questions about how they are generated and propagate in the interstellar medium. Their optical spectra are characterised by emission lines from atomic species which are collisionally excited in the shock waves generated by the interaction of the ejected material with the interstellar medium or previously ejected jet material. These lines contain a wealth of information on jet and shock properties. Beyond the jet/shock morphology and kinematics, information on the gas physical conditions can be derived by developing a grid of shock models the predictions of which are compared with the observed line ratios (e.g., Raga \& Böhm 1986; Hartigan et al. 1987, 1994); or, alternatively, by using spectral diagnos-

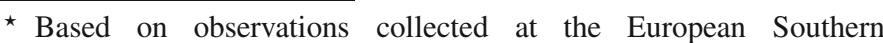
Observatory, La Silla, Chile (ESO programmes 60.C-0398(A).

$\star \star$ Appendix A is only available in electronic form at http://www. aanda.org
}

tics techniques which are independent of any assumption of the heating mechanism (e.g., Bacciotti \& Eislöffel 1999, hereafter referred to as the BE technique). The application of these diagnostic techniques to various datasets allowed derivation of the jet physical/dynamical structure, highlighting the stratification in the shock cooling regions (Nisini et al. 2005; Podio et al. 2006), the variation of the excitation conditions along the jet (Bacciotti \& Eislöffel 1999), across it (Bacciotti et al. 2000; Hartigan \& Morse 2007) and, in a few cases, with gas velocity (Lavalley-Fouquet et al. 2000; Coffey et al. 2008; Garcia Lopez et al. 2008).

It is also important to estimate the dust content in jets, a topic that has been poorly studied to date. Several theoretical studies have explored the dust content and its distribution in the interstellar medium. On the one hand both observations and theoretical models show that the gas-phase abundances of elements like iron $(\mathrm{Fe})$, magnesium $(\mathrm{Mg})$, silicon $(\mathrm{Si})$, and calcium $(\mathrm{Ca})$ are considerably depleted (by a factor of the order of $10^{2}-10^{4}$ ) with respect to their solar abundances because of the formation 
of dust grains (Baldwin et al. 1991; van Dishoek et al. 1993; Savage \& Sembach 1996). On the other hand the chemical composition, the size and the content of dust grains can evolve as they lose atoms to the gas phase through shocks waves. The high energy gas-grain and grain-grain collisions occurring behind the shock front, in fact, may lead to the erosion of the grain surfaces (sputtering) and/or to the vaporisation and fragmentation of grains (Jones et al. 1994; Jones 2000; Draine 2003; Guillet et al. 2009, and references therein). Total dust destruction is expected in high velocity shocks such as supernova-generated shock waves $\left(v_{\mathrm{s}}>200 \mathrm{~km} \mathrm{~s}^{-1}\right)$ but there are very few observations about dust reprocessing in slower shocks such as those occurring along stellar jets $\left(v_{\mathrm{s}} \sim 10-80 \mathrm{~km} \mathrm{~s}^{-1}\right.$ in the jet). A few studies have investigated the gas phase abundance of $\mathrm{Fe}$ in HH jets (Beck-Winchatz et al. 1994, 1996; Mouri \& Taniguchi 2000; Böhm \& Matt 2001; Nisini et al. 2002) and only recently, the analysis has been extended to other refractory species such as $\mathrm{Si}, \mathrm{Ca}$, carbon $(\mathrm{C})$, nickel $(\mathrm{Ni})$, chromium $(\mathrm{Cr})$, and titanium (Ti) (Nisini et al. 2005, 2007; Podio et al. 2006; Garcia Lopez et al. 2008).

To further investigate this topic, we study here the distribution of physical parameters and, in particular, the gas phase abundance of calcium in a sample of $\mathrm{HH}$ jets $(\mathrm{HH} 111, \mathrm{HH} 1 / 2$, $\mathrm{HH} 83, \mathrm{HH} 24 \mathrm{M} / \mathrm{A} / \mathrm{C} / \mathrm{E}, \mathrm{Sz68}$ ), as a function of distance from the source, i.e. in the different working surfaces along the jet, and, where possible, of gas velocity. As we will show, these kind of estimates are very important to understand dust reprocessing in shocks and are also useful to derive constraints on the properties of the jet launching region.

This paper is organized as follows: in Sect. 2 we present the observations and the data reduction process and in Sect. 3 we briefly recall the principle of the so-called BE diagnostic technique, which is used to derive gas physical conditions along the jets. In Sect. 4 we present the physical properties derived for $\mathrm{HH} 111$, in two velocity intervals, and for the jet from Sz68, that is analysed here for the first time. Similar results derived for the other jets in the sample, at higher spatial sampling than in previous studies, are presented in the online material. In Sect. 5 we use the derived physical parameters to estimate the presence of dust grains in the jets and we discuss the efficiency of shocks in reprocessing the dust. A comparison of our results with the predictions of theroretical models is then made. Finally, in Sect. 6 we present a summary of our results.

\section{Observations and data reduction}

We obtained optical spectra of a sample of HH jets (HH 111, HH 1/2, HH 83, HH 24 M/A/C/E, and Sz68). Our spectra were acquired in January 1998 at the ESO 3.6 m-telescope by using the EFOSC2 spectrograph, the GR9 grism and a 1."5 slit width. The slit was aligned parallel to the jet axis with position angles: $276.7^{\circ}$ for $\mathrm{HH} 111,324.7^{\circ}$ for $\mathrm{HH} 1 / 2,297.7^{\circ}$ for $\mathrm{HH} 83,328.1^{\circ}$ for $\mathrm{HH} 24 \mathrm{C} / \mathrm{E}, 219.0^{\circ}$ for $\mathrm{HH} 24 \mathrm{G}$, and $334.9^{\circ}$ for Sz68. The spectra cover the wavelength range from 5600 to $7335 \AA$ with a spectral resolution $\sim 1300\left(F W H M_{\text {instr }} \sim\right.$ $\left.230 \mathrm{~km} \mathrm{~s}^{-1}\right)$. The spatial and spectral sampling scales are $0.157^{\prime \prime} /$ pixel and $0.872 \AA /$ pixel corresponding to $\sim 40 \mathrm{~km} \mathrm{~s}^{-1}$.

The spectral images were flat-fielded, sky-subtracted, wavelength and flux calibrated. In addition to the standard data reduction process the spectral images of the detected lines ([O I] $\lambda \lambda 6300,6363,[\mathrm{~N}$ II] $\lambda \lambda 6548,6583$, [S II] $\lambda \lambda 6716,6731$, and $[\mathrm{Ca}$ II] $] \lambda 7291,7324)$ were velocity-calibrated, resampled to the same velocity scale for all the lines, and corrected for velocity shifts due to atmospheric differential refraction. During the observations, in fact, the slit was aligned along the jet emission through pre-imaging in the [S II] 66731 line. Because of atmospheric differential refraction, however, the peak of the emission in the other lines may be off the slit centre inducing a velocity shift in these lines (see the Appendix of Bacciotti et al. 2002, for a discussion of the uneven slit illumination effect). The resampling in velocity and the corrections mentioned above allowed us to accurately align the lines in velocity despite the low spectral resolution.

As shown in Hartigan \& Morse (2007), the interplay between the slit width and the projected width of the target is crucial in spectroscopic observations. If the slit is narrower than the jet (and the lines are broader than the spectral resolution) the detected lines are spectrally resolved and the velocity resolution is higher for decreasing slit width. If, in contrast, the slit is much wider than the jet (and the velocity dispersion is lower than the spectral resolution) the observations produce emission-line images for each line in the spectrum, but no velocity information can be recovered. The latter is so-called "slitless spectroscopy" (see also Hartigan et al. 2004).

Our observations are seeing-limited and the jet widths are spatially unresolved. Thus the critical parameter to be compared with the slit width is the full width half maximum $(F W H M)$ of the seeing. This varied between $\sim 1^{\prime \prime}$, when observing $\mathrm{HH} 1 / 2$, $\mathrm{HH}$ 83, HH $24 \mathrm{M} / \mathrm{A} / \mathrm{C} / \mathrm{E}$, and Sz68, and $~ 11^{\prime \prime} 5$ when observing HH 111.

In the case of $\mathrm{HH} \mathrm{111,} \mathrm{the} \mathrm{seeing} \mathrm{was} \mathrm{comparable} \mathrm{to} \mathrm{the}$ slit width and the acquired spectra show line profiles which are broader than the spectral resolution. Thus the HH 111 jet is partially resolved in velocity, allowing an analysis of its structure along the jet and in two different velocity intervals, as will be explained in more detail in the next section.

In the other jets, in contrast, the slit is wider than the seeing $F W H M$. Thus we obtain images of the jet in the different lines. This is evident when comparing spectral images of the detected lines with pre-imaging acquired with the [S II] filter. To avoid confusion between the spectral and spatial information we integrated the line fluxes over the line "spectral" profile, suppressing the velocity dimension.

In the next section we explain in more detail the analysis performed in the two different cases.

\section{Application of spectral diagnostic}

Our analysis relies upon the dependence of the ratios between optical forbidden lines on the gas physical conditions in the jet, i.e. on the electron and total hydrogen density, $n_{\mathrm{e}}$ and $n_{\mathrm{H}}$, the hydrogen ionisation fraction, $x_{\mathrm{e}}$, and the electron temperature, $T_{\mathrm{e}}$ (Bacciotti et al. 1995; Bacciotti \& Eislöffel 1999).

As a first step, we thus computed for all the jets in our sample the line ratios used in the diagnostics, i.e. [S II] $\lambda 6731 / 6716,[\mathrm{~N} \mathrm{II}] \lambda \lambda 6548,6583 /[\mathrm{OI}] \lambda \lambda 6300,6363$, and [OI] $\lambda \lambda 6300,6363 /[\mathrm{S} \mathrm{II}] \lambda \lambda 6716,6731$. For HH 111, which is partially spectrally resolved, we divided the spectral images of the detected $\mathrm{S}^{+}, \mathrm{O}^{0}$, and $\mathrm{N}^{+}$lines pixel by pixel, thus obtaining position-velocity (PV) diagrams of the line ratios (see Fig. 1). For the other jets (HH 1/2, HH 83, HH $24 \mathrm{M} / \mathrm{A} / \mathrm{C} / \mathrm{E}$, and Sz68) we integrated over the spectral profile obtaining the variation of the ratios only as a function of the distance from the source. The distribution of the ratios already gives a qualitative idea of the variations of the gas physical conditions along the jet and, for $\mathrm{HH} 111$, with the gas velocity. The ratio between the sulphur doublet lines ([S II] $66731 / 6716$ ) is a tracer of the 

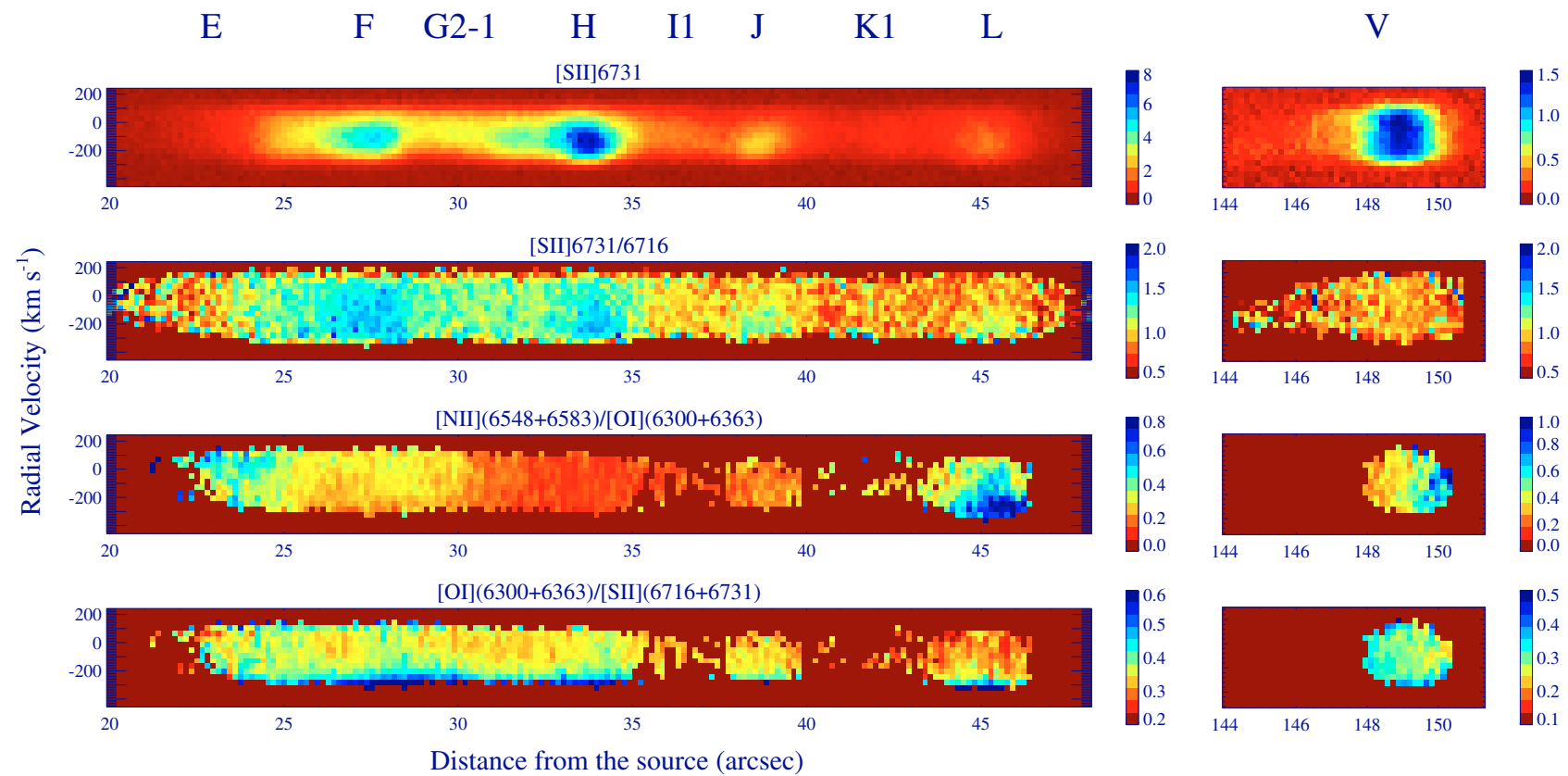

Fig. 1. Position-velocity diagrams of the forbidden line ratios used in the diagnostic technique for the HH 111 jet. From top to bottom panel: the [S II] $] 6731$ line intensity; the $[\mathrm{S} \mathrm{II}] \lambda 6731 / 6716$ ratio increases with electron density, $n_{\mathrm{e}}$; the $[\mathrm{N} \mathrm{II}] /[\mathrm{O} \mathrm{I}]$ ratio mainly depends on the ionisation fraction, $x_{\mathrm{e}}$, and increases for increasing $x_{\mathrm{e}}$; the $[\mathrm{O} \mathrm{I}] /\left[\mathrm{S}\right.$ II] ratio increases for increasing temperature, $T_{\mathrm{e}}$.

gas electron density up to the critical density of the [S II] lines $\left(n_{\mathrm{e}} \sim 2.5 \times 10^{4} \mathrm{~cm}^{-3}\right)$, the $[\mathrm{N} \mathrm{II}] /[\mathrm{O} \mathrm{I}]$ increases primarily with $x_{\mathrm{e}}$, and the $[\mathrm{O} \mathrm{I}] /[\mathrm{S} \mathrm{II}]$ ratio is dependent on both $x_{\mathrm{e}}$ and $T_{\mathrm{e}}$.

To obtain a more quantitative estimate of the gas physical properties we use the BE technique (Bacciotti \& Eislöffel 1999), which extracts diagnostic information from the comparison of observed and calculated ratios. The method is based on the fact that the observed forbidden lines are collisionally excited and that, in low-excitation conditions, and provided that no strong sources of ionizing photons are present, the ionization fraction of hydrogen is tightly related to those of nitrogen and oxygen via charge exchange processes. This allows one to easily retrieve $x_{\mathrm{e}}$ (and the total hydrogen density) and $T_{\mathrm{e}}$. As discussed in Bacciotti \& Eislöffel (1999) this method assumes that the lines used in the technique are emitted in a region of similar temperature, density and ionization fraction. Bacciotti \& Eislöffel (1999) and Nisini et al. (2005) carefully verified this assumption by using the results from shock models (e.g., Hartigan et al. 1987). They showed that the considered forbidden lines have their peak emission in the same region behind shock fronts of different shock velocity, and verified that the obtained parameters are representative averages.

In this paper we use an improved version of the BE code in which the values of the collision strength are updated using the results in Keenan et al. (1996) for $\mathrm{S}^{+}$, Hudson \& Bell (2005) for $\mathrm{N}^{+}$, and Berrington \& Burke (1981) and Mendoza (1983) for $\mathrm{O}^{0}$. This allows us to obtain a better fit of the collision strength for high temperatures $\left(T_{\mathrm{e}}\right.$ between $\left.2-5 \times 10^{4} \mathrm{~K}\right)$ and thus to trace correctly the plasma physical conditions in regions of high excitation, i.e. where the [N II] emission is comparable to that in the [S II] and [O I] lines. Examples of the latter are some knots of the $\mathrm{HH} 83$ and the $\mathrm{HH} 24$ jets where we could not obtain results in our previous study (Podio et al. 2006).

Note that the diagnostic technique uses ratios between different species and, thus, the obtained $x_{\mathrm{e}}$ and $T_{\mathrm{e}}$ may depend on the chosen set of elemental abundances. However, as stressed in Podio et al. (2006), the relative variations of the parameters (i.e. in different knots and/or velocity intervals) do not depend on the choice of an abundance set (see Fig. 1 of Podio et al. 2006) which, therefore, can be thought as a model parameter. On the other hand the most recent determinations by Asplund et al. (2005) (solar) and Esteban et al. (2004) (Orion) are in good agreement and give rise to diagnostic results that differ by at most $15 \%$. One of the main goal of this paper is the determination of the calcium gas-phase abundance with respect to solar. Thus, in contrast with Podio et al. (2006), the solar abundances estimated by Asplund et al. (2005) are adopted for consistency in all our diagnostic analysis. In particular, the values of the temperature and the ionization fraction are larger when using the abundances from Esteban et al. (2004). The uncertainty over the absolute values is not shown in Figs. 3, 4, A.1, A.3, and A.4, because these are intended to be used to analyse the relative variations of parameters along the jet and in different velocity intervals. However, we do consider the uncertainty due to abundances choice when computing theoretical $[\mathrm{Ca}$ II] $] /[\mathrm{S} \mathrm{II}]$ ratios to estimate the calcium gas-phase abundance (see Sect. 5 for more details).

Since the observations are seeing-limited, i.e. the angular resolution of the data is much wider than the spatial sampling, we integrated the line fluxes over the seeing $F W H M$ ( $F W H M_{\text {seeing }} \sim 1.2^{\prime \prime} \sim 8$ pixels) before applying the diagnostics. Thus the de facto spatial sampling in all the figures is equal to the seeing. Moreover, we integrated the line fluxes over the spectral profile for the spectrally unresolved jets (HH 1/2, HH 83, HH 24 $\mathrm{M} / \mathrm{A} / \mathrm{C} / \mathrm{E}, \mathrm{Sz68}$ ) and over two velocity intervals for $\mathrm{HH}$ 111: low (for $v>-100 \mathrm{~km} \mathrm{~s}^{-1}$ ) and high (for $v<-100 \mathrm{~km} \mathrm{~s}^{-1}$ ) velocity Interval (LVI and HVI, respectively).

For the HH 111 and $\mathrm{HH} 1$ jets we corrected the observed fluxes for reddening using the values of the visual extinction, $A_{\mathrm{V}}$, estimated by Nisini et al. (2005) and Podio et al. (2006), the standard dereddening procedure from Draine (1989) and an interpolation of the extinction law derived by Rieke \& Lebofsky (1985) 
for the near-IR bands. For the HH 83 and HH 24 jets we have no estimates of the visual extinction because the [Fe II] $\lambda 1.64,1.32$, 1.25 lines were not detected in our earlier optical/NIR spectra (Podio et al. 2006). Thus we could not correct the line fluxes for the reddening before computing the ratios and applying the diagnostics technique. On the other hand, Bacciotti \& Eislöffel (1999) showed that, since the lines used in this diagnostic are very close in wavelength, any errors induced by not correcting for extinction are at most $8-10 \%$ for the ionization fraction and $15 \%$ for the temperature as long as the visual extinction is lower than $\sim 3$.

Once a set of abundances is assumed, the errors affecting the parameters obtained using the $\mathrm{BE}$ technique are due to the signal-to-noise ratio of the measured fluxes and the uncertainty in the determination of the $A_{\mathrm{V}}$. These are very low $(<5 \%)$ in the bright knots of the $\mathrm{HH} 111, \mathrm{HH} 1$, and $\mathrm{HH} 83$ jets which have strong emission in all the lines, and larger (up to 50\%) in the fainter knots of the HH 24 and Sz68 jets.

\section{The physical structure of the jets}

In this section we briefly describe the new information gathered by applying spectral diagnostics (i.e. the $\mathrm{BE}$ technique) to our $3.6 \mathrm{~m} / \mathrm{EFOSC} 2$ spectra of a sample of HH jets.

In Sect. 4.1 we present the physical parameters $\left(n_{\mathrm{e}}, x_{\mathrm{e}}, T_{\mathrm{e}}\right.$, $\left.n_{\mathrm{H}}\right)$ for $\mathrm{HH} 111$, for which we have been able to highlight for the first time the stratification of the gas physical conditions as a function of velocity.

In Sect. 4.2 we present the parameters obtained for the $\mathrm{HH}$ jet from Sz68 which has not been analysed before with the BE method.

The other objects in our sample, already analysed previously (Bacciotti \& Eislöffel 1999; Nisini et al. 2005; Podio et al. 2006), are here re-analysed with the improved version of the code and increased spatial sampling, for an accurate determination of Ca gas-phase abundance along the jets (see Sect. 5). The physical properties of these jets are shown in the online material, for reference.

\subsection{HH 111: the high and low velocity intervals}

HH 111 is a well-known parsec-scale jet powered by the young star IRAS 05491+0247 located in the L1617 cloud in Orion $(D=460 \mathrm{pc})$. The blueshifted lobe is detected in the optical only from a distance of $\sim 20^{\prime \prime}$ from the source, because the first part is almost completely obscured by a dusty molecular envelope. Then it emerges from the cloud showing a chain of bright, equally spaced and well-collimated knots up to a distance of $\sim 48^{\prime \prime}$ (knots E-L, following the nomenclature from Reipurth et al. 1997). These are followed by a series of more widely spaced and fainter knots up to $80^{\prime \prime}(\mathrm{M}, \mathrm{N}, \mathrm{O}, \mathrm{P})$, and then the jet terminates with a strong bow-shock located at $\sim 150^{\prime \prime}$ (knot V). The physical properties of HH 111 have been investigated by several authors using both optical (e.g., Morse et al. 1993a; Hartigan et al. 1994) and near-infrared (e.g., Nisini et al. 2002) lines. Reipurth et al. (1997) and Davis et al. (2001), instead, focused on the gas kinematics, through analysing [S II] and $\mathrm{H}_{2}$ line profiles, and highlighted the presence of two velocity components peaking at $\sim-75 /-85$ and $\sim-15 /-30 \mathrm{~km} \mathrm{~s}^{-1}$. In a previous work (Podio et al. 2006) a detailed analysis of HH 111 was performed using several spectral tracers, demonstrating the density and temperature stratification in each spatially unresolved cooling region. Nevertheless, none of the previous studies

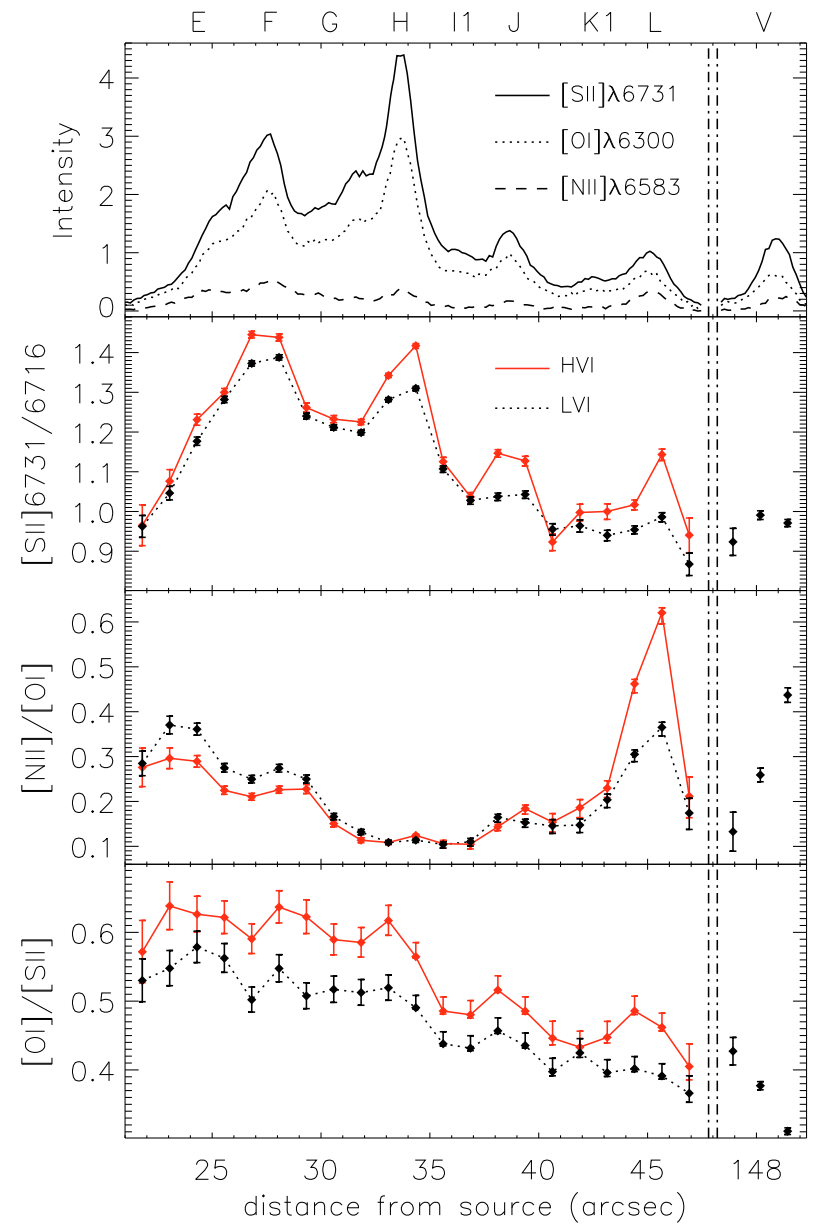

Fig. 2. Variation of the forbidden line ratios used in the diagnostic technique for the $\mathrm{HH} 111$ jet as a function of distance from the source and in two velocity intervals: the high velocity interval (HVI, red points/solid line, $v<-100 \mathrm{~km} \mathrm{~s}^{-1}$ ) and the low velocity interval (LVI, black points/dotted line, $v>-100 \mathrm{~km} \mathrm{~s}^{-1}$ ). In contrast, in the terminal bow (knot V) the ratios have been computed by integrating the fluxes over the line spectral profile thus there is no information on the velocity intervals. From top to bottom panel: intensity profiles of the optical lines, [S II] $]$ 6731/6716 ratios, [N II]/[O I] ratios, and [O I]/[S II] ratios.

investigated the variation of the physical parameters as a function of gas velocity, i.e. in different velocity intervals.

As explained in Sect. 2 the data for HH 111 show partially velocity resolved line profiles. This is confirmed by the positionvelocity (PV) diagrams of the computed line ratios in Fig. 1, which clearly show different value of the ratios in the two velocity intervals in the jet (knots $\mathrm{E}$ to $\mathrm{L}$ ). A comparison of the values of the ratios integrated over the two considered velocity intervals (LVI and HVI) is also shown in Fig. 2. Since these ratios depend on the gas physical conditions, they give a qualitative idea of the variations of electron density, temperature, and ionisation fraction along the jet and with the gas velocity. The PV diagrams of the ratio between the sulphur doublet lines ([S II] $] 6731 / 6716$ ), for example, shows that $n_{\mathrm{e}}$ peaks at the positions of the brightest knots and at high velocities. The [N II]/[O I] and $[\mathrm{OI}] /[\mathrm{S} \mathrm{II}]$ ratios, on the other hand, indicate a peak of the excitation conditions $\left(x_{\mathrm{e}}, T_{\mathrm{e}}\right)$ in the high velocity interval of knot L. Interestingly, HST high-angular resolution images of the jet clearly show that HH 111 is a chain of shock working surfaces where knot L has the clearest "bow" morphology (Reipurth et al. 1997 ) and is the knot with the largest [S II] and $\mathrm{H}_{2}$ line profiles 


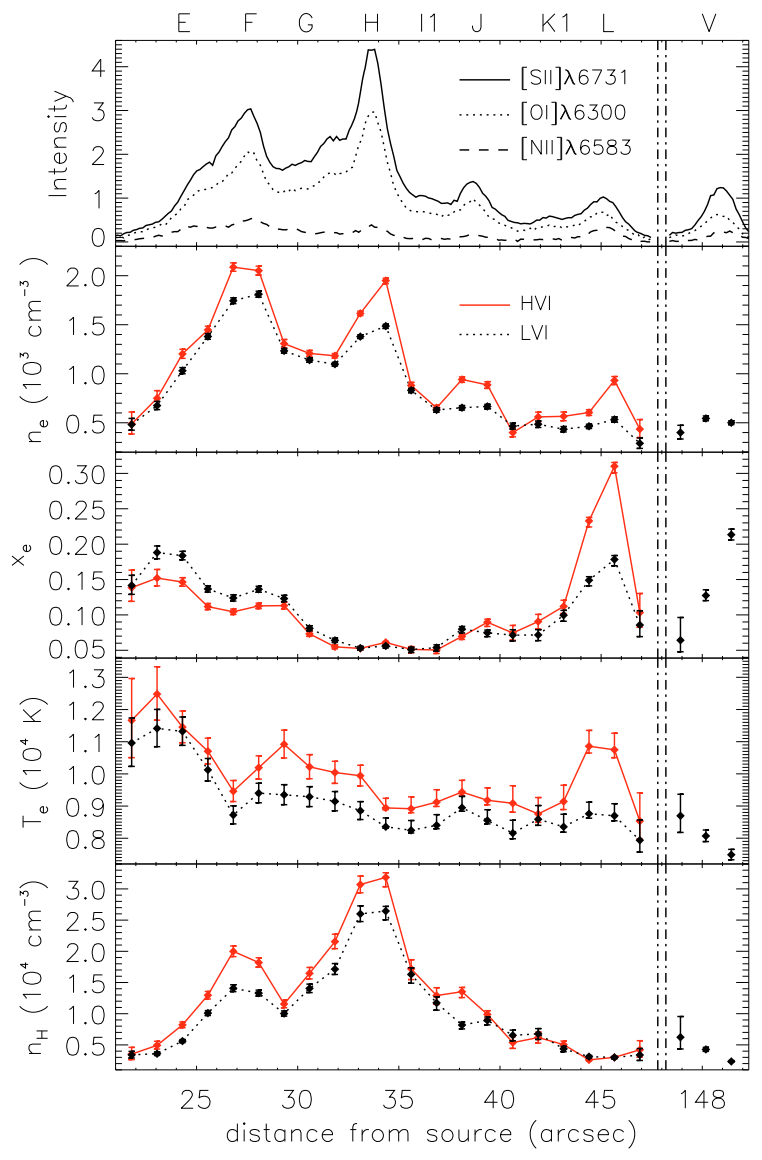

Fig. 3. Variation of the physical parameters for the HH 111 jet as a function of distance from the source and in two velocity intervals: the high velocity interval (HVI, red points/solid line, $v<-100 \mathrm{~km} \mathrm{~s}^{-1}$ ) and the low velocity interval (LVI, black points/dotted line, $v>-100 \mathrm{~km} \mathrm{~s}^{-1}$ ). In contrast, in the terminal bow (knot $\mathrm{V}$ ) the parameters are derived by integrating the fluxes over the line spectral profile thus there is no information on the velocity intervals. From top to bottom panel: intensity profiles of the optical lines, the electron density, $n_{\mathrm{e}}$, in units of $10^{3} \mathrm{~cm}^{-3}$, the ionisation fraction, $x_{\mathrm{e}}$, the temperature, $T_{\mathrm{e}}$, in units of $10^{4} \mathrm{~K}$, and the total density, $n_{\mathrm{H}}$, in units of $10^{4} \mathrm{~cm}^{-3}$.

(Reipurth et al. 1997; Davis et al. 2001) and the largest shock velocity (Hartigan et al. 2001) in the jet beam. Our PV diagrams confirm this picture highlighting the shock velocity structure.

By integrating the line fluxes over the seeing FWHM and over the LVI and HVI and then by applying the BE technique we obtain the variations of the physical parameters both along the jet and in the two velocity intervals for knots E-L (see Fig. 3). The derived parameters are in agreement with previous estimates (Podio et al. 2006): $n_{\mathrm{e}}$ varies between $10^{2}$ and $\sim 2.2 \times 10^{3} \mathrm{~cm}^{-3}$, $x_{\mathrm{e}}$ goes from a few percent to approximately $30 \%, T_{\mathrm{e}} \sim 0.7-$ $1.3 \times 10^{4} \mathrm{~K}$, and $n_{\mathrm{H}}$ ranges between $\sim 2 \times 10^{3}$ and $\sim 3 \times 10^{4} \mathrm{~cm}^{-3}$. With respect to previous analyses, however, the increased spatial sampling (more than twice that of previous studies) and the velocity information highlight several shocks features, such as $n_{\mathrm{e}}$ maxima in the HVI of the brightest knots, higher values of $T_{\mathrm{e}}$ in the HVI along the full jet length, and, finally, a strong and steep increase of $x_{\mathrm{e}}$ and $T_{\mathrm{e}}$ in the HVI of knot L. Note that very weak emission in the [O III] $\lambda \lambda 44959,5007$ lines was detected by Morse et al. (1993a) in this knot. The presence of $\mathrm{O}^{++}$is not accounted for in the adopted diagnostic technique. Nevertheless, since the emission in these lines is very weak, we tentatively applied the diagnostic to knot $\mathrm{L}$ and the obtained high values of $x_{\mathrm{e}}$ and $T_{\mathrm{e}}$ are in agreement with the detection of such high-excitation lines. These results are an important observational validation of the shock velocity structure predicted by models (Hartigan et al. 1987).

We do not apply the diagnostic to knots $\mathrm{M}, \mathrm{N}, \mathrm{O}$, and $\mathrm{P}$ because of the very weak emission in the [N II] lines. Nevertheless, the electron density can be inferred by integrating the [S II] emission over the knot spatial profile to increase the $\mathrm{S} / \mathrm{N}$, which is low at the position of these faint knots. We obtain $n_{\mathrm{e}}$ values of about $150 \mathrm{~cm}^{-3}, 200 \mathrm{~cm}^{-3}, 250 \mathrm{~cm}^{-3}$, and $150 \mathrm{~cm}^{-3}$ in the HVI of knot M, N, O, and P and values of $\sim 100 \mathrm{~cm}^{-3}$ lower in the LVI for the same knots. The average values are in agreement with previous results from Podio et al. (2006).

Previous studies did not detect high excitation lines at the position of the terminal bow shock (knot V) (Morse et al. 1993b), thus we apply our diagnostic to this knot. Even if two velocity components were detected in [S II] by Reipurth et al. (1997) the PVs in Fig. 1 do not show different values of the ratios in the two velocity intervals. This can be due to the low spectral resolution combined with the much lower intensity of knot $\mathrm{V}$ with respect to other knots in the jet (knot E-L). Thus we integrate the line fluxes over their spectral profiles obtaining the trend shown in Fig. 3. The electron and total density are $\sim 400-550 \mathrm{~cm}^{-3}$ and $\sim 2-5 \times 10^{3} \mathrm{~cm}^{-3}$, respectively. The ionisation fraction and the temperature show an unexpected anti-correlation and varies between $\sim 0.23$ and $\sim 0.07$, and $\sim 0.75 \times 10^{4} \mathrm{~K}$ and $\sim 0.85 \times 10^{4} \mathrm{~K}$ moving from the shock front towards the source. Note that despite the high shock velocity of knot V (Hartigan et al. 2001) we did not infer high values of $x_{\mathrm{e}}$ and $T_{\mathrm{e}}$ as for knot $\mathrm{L}$.

\subsection{The Sz68 jet}

The Sz68 jet has never been analysed using spectral diagnostic techniques. This jet is located in the B228 molecular cloud between the two emission-line stars Sz68 and Sz69. The collimated chain of [S II] shock-excited blueshifted emission, extending up to $34^{\prime \prime}$ from Sz68 at a position angle of $135^{\circ}$, was detected by Heyer \& Graham (1989). They report three knots located at 21", $28^{\prime \prime}$, and $34^{\prime \prime}$ from the source, the brightest ones being the first knot, A, and the last one, B. Comparing the position of the knots in the spectra of Heyer \& Graham (1989) with the positions in our EFOSC2 data (see upper panel of Fig. 4 for the knots spatial profile), we derive an estimate of the knot proper motions. This indicates a tangential velocity of $\sim 600 \mathrm{~km} \mathrm{~s}^{-1}$ for knot A, $\sim 460 \mathrm{~km} \mathrm{~s}^{-1}$ for the intermediate knot, and $\sim 330 \mathrm{~km} \mathrm{~s}^{-1}$ for knot $\mathrm{B}$, which combined with the radial velocity corrected for the source heliocentric velocity $\left(\sim-55 \mathrm{~km} \mathrm{~s}^{-1}\right.$ for knot A and $\sim-29 \mathrm{~km} \mathrm{~s}^{-1}$ for knot B, Heyer \& Graham 1989) gives an upper limit for the inclination angle out of the plane of the sky of $\sim 5^{\circ}$.

We applied the BE technique to the line fluxes measured along the three knots by integrating over the line spectral profiles. Since this object is very faint, the error-bars are much larger than for the other jets. The inferred electron density is quite low varying between $200 \mathrm{~cm}^{-3}$ and $1.1 \times 10^{3} \mathrm{~cm}^{-3}$. These values are slightly lower than those derived by Heyer \& Graham (1989). The ionisation fraction is $0.05-0.25$ and the temperature ranges between $0.6-1.1 \times 10^{4} \mathrm{~K}$, with a maximum at the position of the intermediate knot, where there is a peak of $n_{\mathrm{e}}$, as well. Finally the hydrogen total density is $2-6 \times 10^{3} \mathrm{~cm}^{-3}$.

The determination of the jet physical conditions, as well as the jet tangential velocity and inclination angle allows us to estimate the mass loss rate as $\dot{M}_{\text {jet }}=\mu m_{\mathrm{H}} n_{\mathrm{H}} \pi r_{J}^{2} v_{J}$, where $\mu=1.41$ is the mean atomic weight, $m_{\mathrm{H}}$ the proton mass, $n_{\mathrm{H}}$ the hydrogen density and $r_{J}$ and $v_{J}$, respectively, the jet radius and velocity. By assuming a typical jet radius of $\sim 100-300$ AU we obtain a mass 


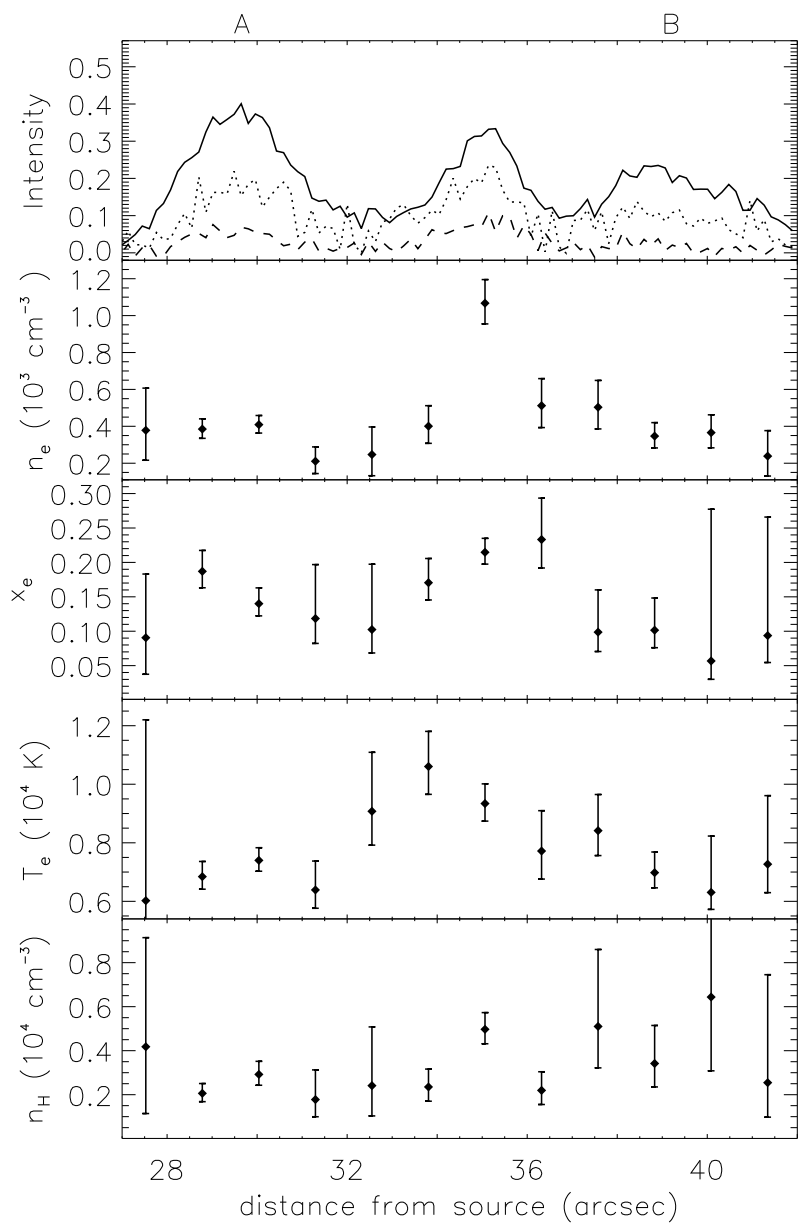

Fig. 4. Variation of the physical parameters for the Sz68 jet as a function of the distance from the source. From top to bottom panel: intensity profiles of the optical lines, the electron density, $n_{\mathrm{e}}$, in units of $10^{3} \mathrm{~cm}^{-3}$, the ionisation fraction, $x_{\mathrm{e}}$, the temperature, $T_{\mathrm{e}}$, in units of $10^{4} \mathrm{~K}$, and the total density, $n_{\mathrm{H}}$, in units of $10^{4} \mathrm{~cm}^{-3}$.

loss rate between a few $10^{-8}$ and a few $10^{-7} M_{\odot} \mathrm{yr}^{-1}$. These values are of the same order of magnitude of $\dot{M}_{\text {jet }}$ found for typical HH jets (Hartigan et al. 1994; Bacciotti \& Eislöffel 1999; Podio et al. 2006).

\section{Dust reprocessing in the jets}

Refractory species, such as $\mathrm{Ca}, \mathrm{Fe}, \mathrm{Ni}, \mathrm{Cr}, \mathrm{Ti}, \mathrm{Mg}$, Si are often depleted in the interstellar medium because their atoms are locked into dust grains (Savage \& Sembach 1996). The calcium gas-phase abundance, for example, is estimated to be $\sim 2 \times 10^{-8}$ in Orion (Baldwin et al. 1991), i.e. two orders of magnitude lower than its solar abundance $\left(\sim 2.04 \times 10^{-6}\right.$, Asplund et al. 2005), and up to 4 orders of magnitude lower than solar in $\zeta$ Oph (Savage $\&$ Sembach 1996). On the other hand, the passage of shocks can partially or completely destroy the dust releasing the refractory atoms into the gas phase.

Theoretical models of dust reprocessing in shocks, such as those driven by supernova explosions or those occurring in stellar jets, estimate the dust destruction rate by considering the different processes at work, i.e. thermal and inertial sputtering, photoevaporation and shattering. They show that the relative importance of these processes, and thus the efficiency of the shock in reprocessing dust, strongly depends on a number of parameters such as the shock velocity, the gas pre-shock density, the intensity of the magnetic field, and the size and structure of the dust grains (e.g., Jones et al. 1994; Jones 2000; Draine 2003; Guillet et al. 2009).

From an observational point of view an estimate of the depletion of refractory species with respect to their solar abundance can be used to gauge the amount of dust grains in jets from young stars and thus to evaluate the efficiency of mild shocks in destroying the dust. This in turn can help constrain the location and size of the region around the star from where the jet originates.

In Sect. 5.1 we explain in detail the method used to estimate the abundance of calcium in the gas-phase, while in Sect. 5.2 we present the results obtained and in Sect. 5.3 we compare them with the predictions of theoretical models.

\subsection{Observational estimates of calcium gas-phase abundance in stellar jets: the method}

To estimate the depletion of a given refractory specie, in our case calcium, with respect to its solar abundance, we compare "expected" and observed ratios between refractory and nonrefractory species known to be present in solar abundance in the interstellar medium (e.g. Savage \& Sembach 1996).

The "expected" ratios can be computed through two different methods:

(i) by using shock models which predict the gas physical conditions and thus the emission in different lines behind the shock front. This method was used by Mouri \& Taniguchi (2000) and, in part, by Nisini et al. (2002) to estimate $\mathrm{Fe}$ gas-phase abundance in $\mathrm{HH}$ jets. They found that $\mathrm{Fe}$ is strongly depleted in these objects, up to $80 \%$ with respect to its solar abundance;

(ii) by using the gas physical conditions inferred through spectral diagnostic tools. In this case the estimate does not depend upon assumptions of the shock characteristics (velocity, pre-shock density, magnetic field intensity) but depends on the accuracy of the determinations of the gas parameters. Beck-Winchatz et al. $(1994,1996)$ used this method to estimate $\mathrm{Fe}$ and $\mathrm{Ni}$ gas-phase abundance in $\mathrm{HH}$ jets. They found that $\mathrm{Fe}$ is not, or only partially, depleted in $\mathrm{HH}$ jets, while $\mathrm{Ni}$ is surprisingly over-abundant. The method was then used by Nisini et al. $(2002,2005)$ and Podio et al. (2006) to estimate the gas-phase abundance of both $\mathrm{Fe}$ and $\mathrm{Ca}$ obtaining a strong depletion (up to 90\%) of these species which, however, may vary along the jet and decreases to $0 \%$ in some knots.

The main assumption of method (ii) is that the observed lines from refractory and non-refractory species are emitted in the same region behind the shock front, in such a way that the filling factor and the gas physical conditions are the same. This has been verified by Nisini et al. (2005) (see their Fig. 3 and corresponding discussion).

Following Nisini et al. (2005) and Podio et al. (2006), here we use the [Ca II] $\lambda 7291 /[\mathrm{S}$ II] 26731 ratio to estimate the Ca gasphase abundance in our sample of $\mathrm{HH}$ jets. These lines have similar excitation temperatures $\left(\sim 2 \times 10^{4} \mathrm{~K}\right.$ for $[\mathrm{Ca}$ II] $\lambda 7291$ and $\sim 2.2 \times 10^{4} \mathrm{~K}$ for $\left.[\mathrm{S} \mathrm{II}] \lambda 6731\right)$ and, despite the critical density of the calcium line being larger than that of the sulphur line $\left(n_{\mathrm{cr}}([\mathrm{S}\right.$ II $]) \sim 2.5 \times 10^{4} \mathrm{~cm}^{-3}, n_{\mathrm{cr}}([\mathrm{Ca}$ II $\left.]) \sim 5 \times 10^{7} \mathrm{~cm}^{-3}\right)$, at the typical low density of $\mathrm{HH}$ jets the emission of the two lines peaks at the same distance from the shock front. In the same 
region the $[\mathrm{S} \mathrm{II}],[\mathrm{OI}]$, and $[\mathrm{N}$ II] lines which are used to derive the gas physical conditions along the jet are also excited. Thus the estimated parameters can be used consistently to model the theoretical $[\mathrm{Ca} \mathrm{II}] /[\mathrm{S}$ II] ratio.

Following Hartigan et al. (2004) we assume that the [Ca II] lines are collisionally excited and compute the level populations. As in previous works (Nisini et al. 2005; Podio et al. 2006) we assume that there is no calcium in the form of $\mathrm{Ca}^{0}$, because its ionization potential is very low, $\sim 6.1 \mathrm{eV}$. On the other hand, the ionization potential of $\mathrm{Ca}$ II is also quite low, $\sim 11.9 \mathrm{eV}$, i.e. lower than that for hydrogen $(\sim 13.6 \mathrm{eV})$. In Nisini et al. (2005) and Podio et al. (2006) we computed the [Ca II]/[S II] ratios by assuming that all calcium is in the form of $\mathrm{Ca}^{+}$. This is a reasonable approximation in the case of poorly ionized objects such as $\mathrm{HH} 1$ and $\mathrm{HH} 111\left(x_{\mathrm{e}} \leq 0.3\right)$. However, the presence of $\mathrm{Ca}$ in the form of $\mathrm{Ca}^{++}$cannot be neglected when analysing higher excitation jets such as $\mathrm{HH} 83$ and $\mathrm{HH} 24$. The coefficients for collisional ionization and radiative and dielectronic recombination of $\mathrm{Ca}^{+}$and $\mathrm{H}^{0}$ are very similar for temperatures between $8 \times 10^{3} \mathrm{~K}$ and $3 \times 10^{4} \mathrm{~K}\left(\right.$ at $T \sim 2 \times 10^{4} \mathrm{~K}$, the recombination rates for $\mathrm{Ca}^{+}$and $\mathrm{H}^{0}$ are: $\alpha\left(\mathrm{Ca}^{+}\right) \sim 3.9 \times 10^{-13} \mathrm{~cm}^{3} \mathrm{~s}^{-1}, \alpha\left(\mathrm{H}^{0}\right) \sim 2.5 \times$ $10^{-13} \mathrm{~cm}^{3} \mathrm{~s}^{-1}$; while the coefficients for collisional ionization are: $\left.\mathrm{C}\left(\mathrm{Ca}^{+}\right) \sim 1.2 \times 10^{-11} \mathrm{~cm}^{3} \mathrm{~s}^{-1}, \mathrm{C}\left(\mathrm{H}^{0}\right) \sim 1.6 \times 10^{-11} \mathrm{~cm}^{3} \mathrm{~s}^{-1}\right)$. Therefore, given that at these temperatures all calcium is in the form of $\mathrm{Ca}^{+}$and $\mathrm{Ca}^{++}$, in conditions of ionization equilibrium the ionization fraction of $\mathrm{Ca}^{+}$(defined as $\left.\mathrm{x}\left(\mathrm{Ca}^{+}\right)=\mathrm{Ca}^{++} / \mathrm{Ca}\right)$ and $\mathrm{H}^{0}$ are almost the same: $x\left(\mathrm{Ca}^{+}\right) \sim x_{\mathrm{e}}$. In stellar jets, however, the ionization fraction of hydrogen is never at equilibrium with the local temperature because, as explained in Bacciotti \& Eislöffel (1999), the gas is moving along the jet and the recombination timescale at the observed electron densities is of $\sim 10^{3} \mathrm{yr}$, i.e. of the same order of the jet dynamical time. Because of the similarity of the recombination and collisional ionization coefficients, however, $\mathrm{Ca}^{+}$and $\mathrm{H}^{0}$ atoms can be thought to be undergoing the same processes when moving along the jet. Thus we assume that, at every position along the jet, the $\mathrm{Ca}^{+}$ionization fraction is equal to the hydrogen ionization fraction, $x_{\mathrm{e}}$. Taking $\mathrm{Ca}$ and $\mathrm{S}$ gas-phase abundances equal to solar ones (Asplund et al. 2005), we can finally compute the $[\mathrm{Ca}$ II $] /[\mathrm{S}$ II] theoretical ratios.

Note that, in contrast with previous works (Nisini et al. 2005; Podio et al. 2006), here we use the [Ca II] $\lambda 7291 /[$ S II $] \lambda 6731$ ratio, instead of the $[\mathrm{CaII}] \lambda(7291+7324) /[\mathrm{S} \mathrm{II}] \lambda(6716+6731)$, since the $[\mathrm{CaII}] \lambda 7324$ line may be blended with the [O II] $] \lambda \lambda 7318.6,7319.4$ and the [O II] $\lambda \lambda 7329.9,7330.7$ lines. As explained in Hartigan et al. (2004) the calcium line ratio [Ca II] $\lambda 7291 / \lambda 7324$ is expected to be $\sim 1.5$ both in the case of collisional excitation and for fluorescent pumping from the ground state. Thus by measuring the ratio between the calcium lines we can check for the presence, if any, of [O II] emission blended with the $[\mathrm{CaII}] \lambda 7324$ line. We find that this is not the case for the HH 1 and the HH 34 jets (Nisini et al. 2005; Podio et al. 2006), but may be the case in the HH 111, HH 83 and HH 24 jets which show higher excitation conditions in some of the knots (see Figs. 3, A.3, and A.4). These jets, in fact, show a wider [Ca II] $\lambda 7324$ line in some knots indicating blending with the [O II] lines. Moreover, in our EFOSC2 spectra, the [Ca II] $\lambda 7324$ line is at the border of the detector and hence the line flux can be affected by distortion and/or lower $\mathrm{S} / \mathrm{N}$. Thus we decided to use the $[\mathrm{Ca}$ II $] \lambda 7291 /[\mathrm{S}$ II $] \lambda 6731$ for all the jets in our sample.

For the HH 111 and the HH 1 jet we corrected the observed $[\mathrm{Ca}$ II $] /[\mathrm{S} \mathrm{II}]$ ratios for reddening following the procedure explained in Sect. 3. For the HH 83 and the HH 24 jet we have no estimates of the visual extinction, thus we could not correct
Table 1. Gas-phase abundance of calcium with respect to solar Abundances.

\begin{tabular}{cc}
\hline \hline Jet & {$[\mathrm{Ca}]_{\text {gas }} /[\mathrm{Ca}]_{\text {solar }^{a}}$} \\
\hline HH 111 & $0.21-0.52$ \\
HH 1 & $0.25-0.79$ \\
HH 83 & $0.53-1.57^{*}$ \\
HH 24 A & $0.40-0.69^{*}$ \\
HH 24 E & $0.62-0.85^{*}$ \\
HH 24 C & $0.52-0.82^{*}$ \\
HH 34 & $0.3-1$ \\
\hline Orion Cloud $^{c}$ & 0.01 \\
\hline
\end{tabular}

${ }^{a}(\mathrm{Ca} / \mathrm{H})$ solar abundance from Asplund et al. (2005): $2.04 \times 10^{-6} ;{ }^{b}$ from Podio et al. (2006); ${ }^{c}(\mathrm{Ca} / \mathrm{H})$ abundance estimated in the interstellar medium in Orion by Baldwin et al. (1991): $2 \times 10^{-8} ;{ }^{*}$ the values of $[\mathrm{Ca}]_{\text {gas }} /[\mathrm{Ca}]_{\text {solar }}$ for HH 83 and HH 24 have been estimated assuming $A_{\mathrm{V}}=0$, thus they are upper limits to the real values.

the $\mathrm{Ca}^{+}$and $\mathrm{S}^{+}$line fluxes for reddening and the observed ratios (computed assuming $A_{\mathrm{V}}=0$ ) are actually upper limits to the real values (the latter decrease for increasing $A_{\mathrm{V}}$ ). This means that the calcium depletion inferred from Figs. 7 and 8 is a lower limit.

The errors affecting the observed ratios are due to the signalto-noise over the measured line fluxes. For HH 1 and HH 111 we consider also the errors due to the uncertainty in estimating $A_{\mathrm{V}}$.

The errors on the theoretical ratios are due to the uncertainty of the estimated physical parameters and are computed by varying $n_{\mathrm{e}}, x_{\mathrm{e}}$, and $T_{\mathrm{e}}$ between their minimum and maximum values. The influence of these errors is represented in the form of a green stripe in Figs. 5-8. Since, as explained in Sect. 3, the choice of the abundance set may affect the absolute values of $x_{\mathrm{e}}$ and $T_{\mathrm{e}}$, we also computed the variations of $[\mathrm{Ca}$ II $] /[\mathrm{S} \mathrm{II}]$ when adopting the alternative abundance set given by Esteban et al. (2004). The difference between the $[\mathrm{Ca} \mathrm{II}] /[\mathrm{S}$ II $]$ ratios obtained with the two sets is illustrated by the width of the beige stripes in Figs. 5-8.

\subsection{Results: Ca depletion along $\mathrm{HH}$ jets}

The comparison between the observed and the predicted $[\mathrm{Ca} \mathrm{II}] /[\mathrm{S} \mathrm{II}]$ ratios is shown in Figs. 5-8 and the inferred values of the calcium gas-phase abundance are summarized in Table 1. Note that $[\mathrm{Ca}]_{\text {gas }} /[\mathrm{Ca}]_{\text {solar }}$ values in Table 1 and in the bottom panel of Fig. 5 are derived by using the $[\mathrm{Ca} I I] /[\mathrm{S} \mathrm{II}]$ ratios predicted assuming solar elemental abundances from Asplund et al. (2005) (illustrated by a dotted line in the figures).

Along the $\mathrm{HH} 111$ and $\mathrm{HH} 1$ jets the observed $[\mathrm{Ca}$ II $] \lambda 7291 /[\mathrm{S} \mathrm{II}] \lambda 6731$ ratios are lower than the predicted ones, i.e. calcium is depleted with respect to its solar abundance, the depletion being between $20 \%$ and $80 \%$. For these jets the results agree with what has been found in Nisini et al. (2005) and Podio et al. (2006), slight differences being explained by the fact that in the latter studies the presence of calcium in the form of $\mathrm{Ca}^{++}$was neglected and a different set of abundances was assumed.

In constrast, along $\mathrm{HH} 83$ dust grains appear to have been destroyed substantially, as the derived $\mathrm{Ca}$ abundance is equal or even larger than solar in the first knots and is depleted up to $47 \%$ at distances larger than $25^{\prime \prime}$ from the source. In the case of $\mathrm{HH} 83$, however, we did not correct the fluxes for extinction, thus the obtained values of $\mathrm{Ca}$ gas-phase abundance are upper limits. It can be shown that with an $A_{\mathrm{V}}=5$ the Ca gas-phase abundance is between 0.36 and 1 . In any case the low depletion in this jet appears to be consistent with its high excitation. 


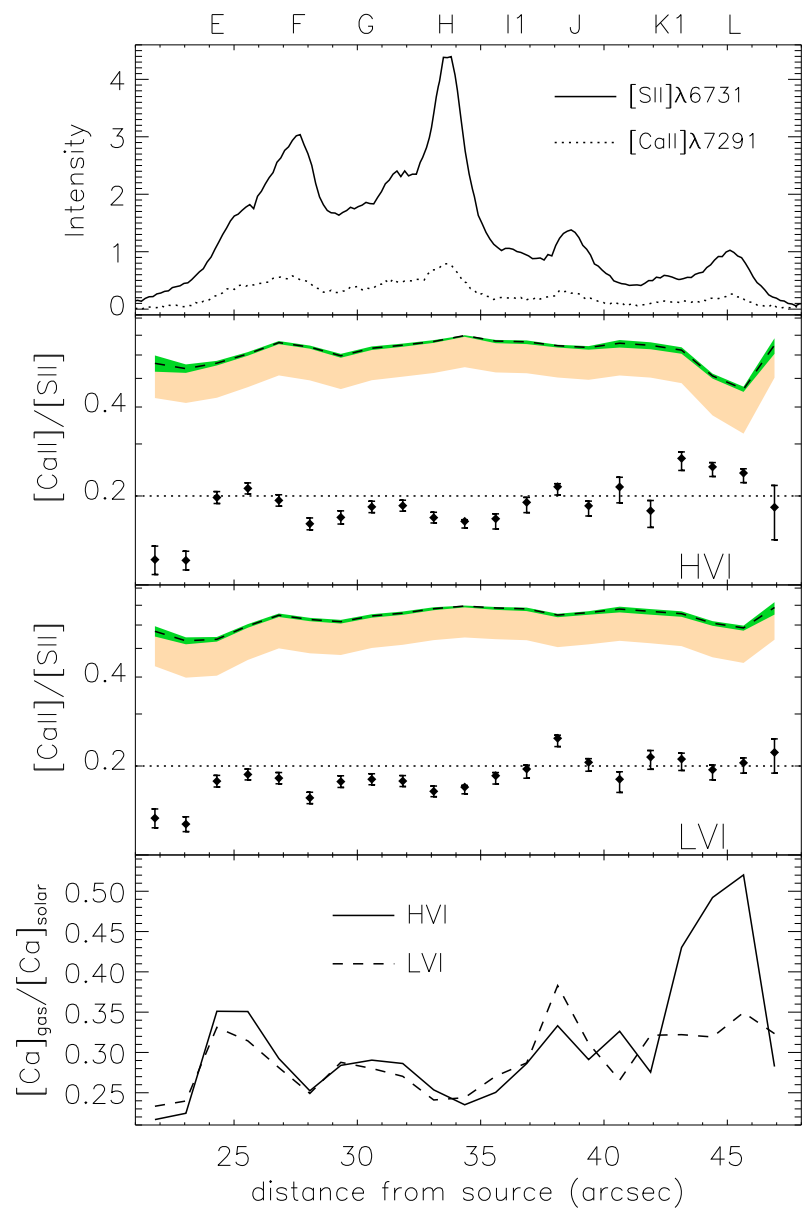

Fig. 5. Comparison between observed (diamonds) and predicted (dashed lines) [Ca II] $\lambda 7291 /[\mathrm{S} \mathrm{II}] \lambda 6731$ along the HH 111 jet and in two velocity intervals: the HVI $\left(v<-100 \mathrm{~km} \mathrm{~s}^{-1}\right.$, second panel $)$ and the LVI $\left(v>-100 \mathrm{~km} \mathrm{~s}^{-1}\right.$, third panel). The dotted line refers to the predicted ratios computed assuming solar abundances from Asplund et al. (2005) and the green upper stripe shows the variation of the ratios due to the errors on the inferred physical parameters. The beige lower stripe, in contrast, shows the variations of the predicted ratios if non-solar abundances from Esteban et al. (2004) are used. In any event, the difference between observed and predicted ratios indicates a depletion of the $\mathrm{Ca}$ gas-phase abundance, which is quantified in the bottom panel using the predicted ratios computed assuming solar abundances (dotted lines).

Finally, in HH 24 we derive the [Ca II] $\lambda 7291 /[\mathrm{S}$ II] $\lambda 6731$ ratio only in a few of the observed knots because for the fainter ones the $\mathrm{Ca}^{+}$emission is below our assumed $\mathrm{S} / \mathrm{N}$ threshold (equal to three times the background noise). Figure 8 shows that the depletion along the jet shows no evident trend: calcium is depleted by $48 \%-18 \%$ in HH $24 \mathrm{C}$, by $~ 38 \%-15 \%$ in HH $24 \mathrm{E}$ and by $\sim 60 \%-31 \%$ in HH 24 A. The lack of a trend along the jet reflects the situation found for the physical parameters (see Fig. A.4) and supports the idea that HH 24 C, E, and A may not belong to a single jet (Eislöffel \& Mundt 1997). Note that also in the case of HH 24 we did not correct the observed [Ca II]/[S II] ratios for extinction thus the inferred $\mathrm{Ca}$ depletion values are lower limits.

The width of the beige stripes in Figs. 5-8 show the variation of the theoretical [Ca II $] /[\mathrm{S} \mathrm{II}]$ ratios when adopting abundances from Esteban et al. (2004). Note that also in this case calcium turns out to be largely depleted in most of the knots, although the inferred $\mathrm{Ca}$ depletion is lower.

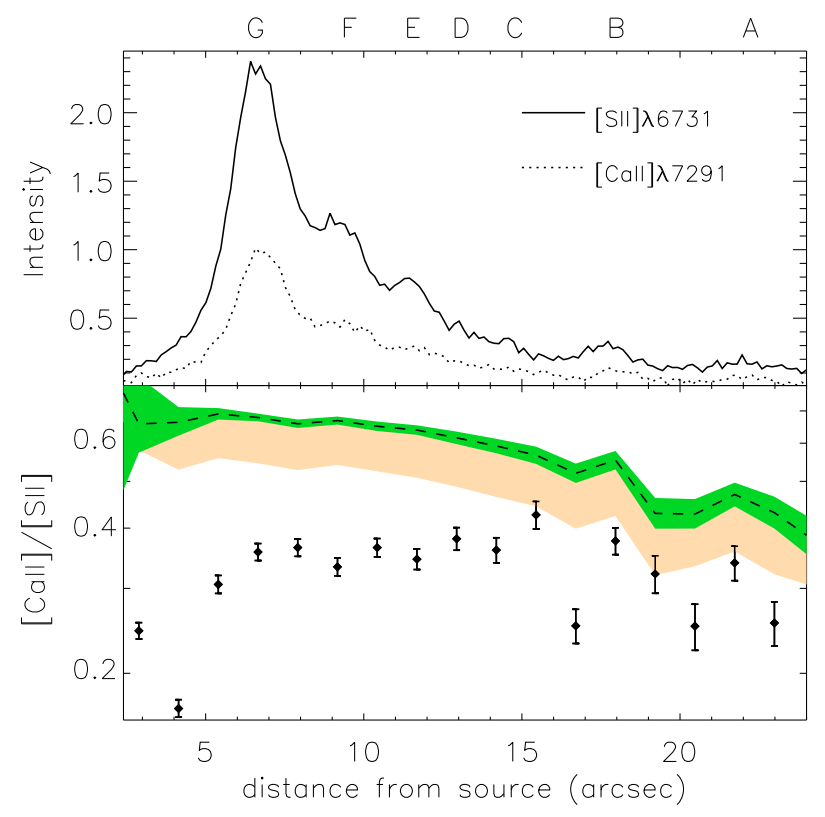

Fig. 6. Comparison between observed (diamonds) and predicted (dashed line) [Ca II] $\lambda 7291 /[\mathrm{S}$ II] $\lambda 6731$ along the HH 1 jet. The green upper and beige lower stripes show the variation of the predicted ratios, as explained in Fig. 5. The difference between observed and predicted ratios indicates a depletion of the $\mathrm{Ca}$ gas-phase abundance.

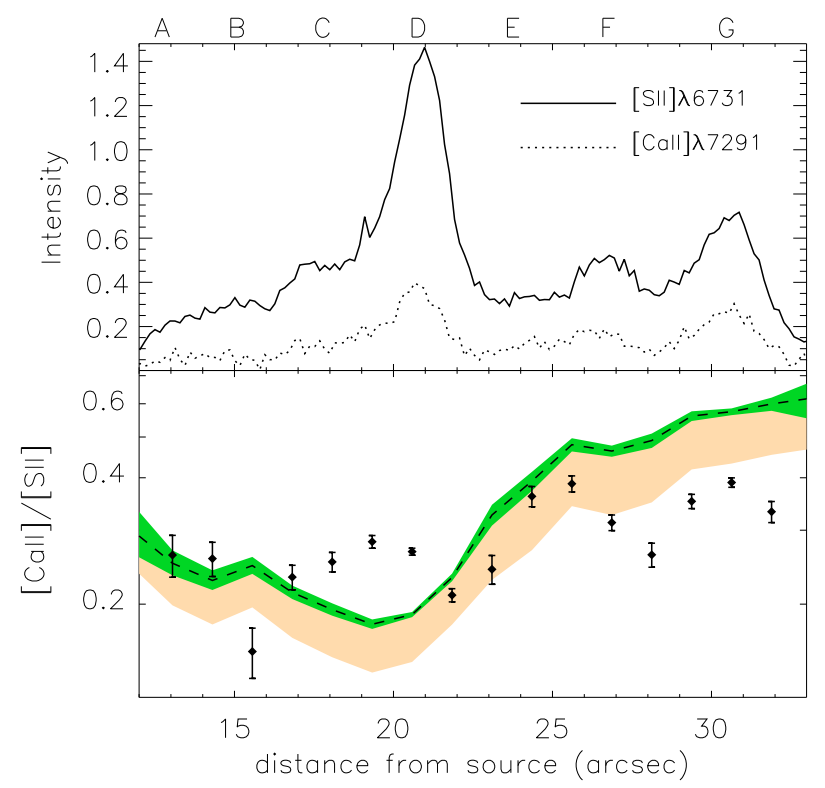

Fig. 7. Comparison between observed (diamonds) and predicted (dashed line) [Ca II] $\lambda 7291 /[\mathrm{S} \mathrm{II}] \lambda 6731$ along the HH 83 jet. The observed ratios are not corrected for reddening $\left(A_{\mathrm{V}}=0\right)$, and are thus upper limits. The green upper and beige lower stripes show the variation of the predicted ratios, as explained in Fig. 5.

\subsection{Discussion}

In general, we find an amount of $\mathrm{Ca}$ depletion comparable to the Fe depletion estimated in other studies (Beck-Winchatz et al. 1996; Böhm \& Matt 2001; Mouri \& Taniguchi 2000; Nisini et al. 2002, 2005; Podio et al. 2006; Garcia Lopez et al. submitted to A\&A). Together the results obtained for the two different refractory species indicate that, despite the presence of shocks, there are still dust grains in the jets. However, partial or total destruction of dust grains can be inferred by comparing the level of 


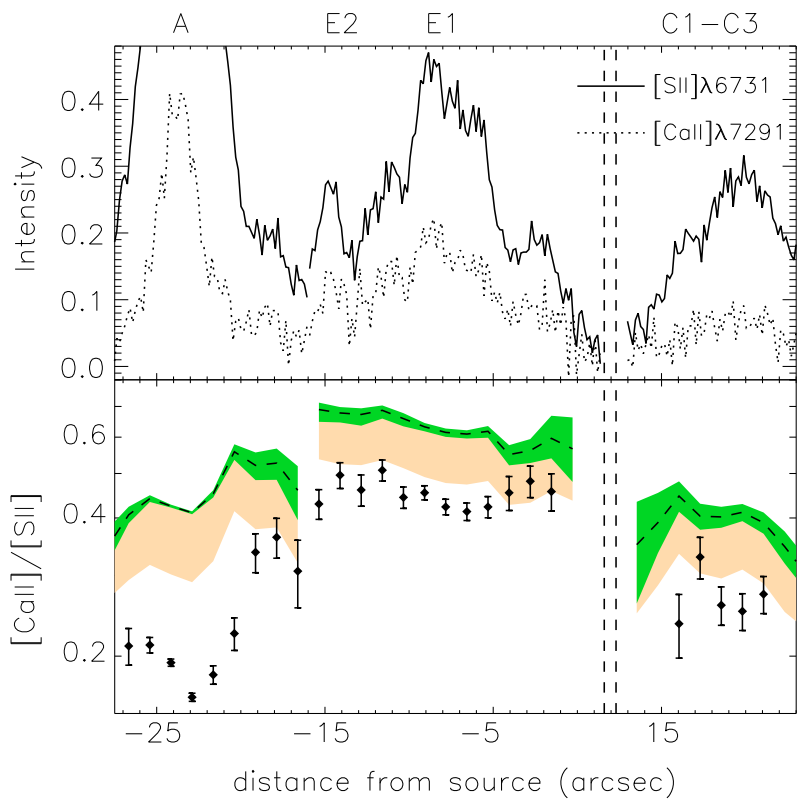

Fig. 8. Comparison between observed (diamonds) and predicted (dashed line) [Ca II] $\lambda 7291 /[\mathrm{S} \mathrm{II}] \lambda 6731$ along the HH 24 jet. The observed ratios are not corrected for reddening $\left(A_{\mathrm{V}}=0\right)$ and thus they are an upper limits. The green upper and beige lower stripes show the variation of the predicted ratios, as explained in Fig. 5.

calcium depletion in the jets with that of the diffuse interstellar medium in Orion (see Table 1).

According to the most recent models of dust reprocessing from Guillet et al. (2009) the dust destruction rate in J-shocks with velocities lower than $50 \mathrm{~km} \mathrm{~s}^{-1}$ and pre-shock densities from $10^{4}$ to $10^{6} \mathrm{~cm}^{-3}$ is around a few percent. Previous models by Jones et al. (1994) predict a destruction rate for silicate grains as high as $50 \%$ for shock velocities of $\sim 100 \mathrm{~km} \mathrm{~s}^{-1}$ and low values of the $b$ parameter ( $b=B_{0} n_{0}^{-1 / 2}$, where $B_{0}$ is the intensity of the magnetic field and $n_{0}$ is the pre-shock density). Finally, up to $90 \%$ of $\mathrm{Fe}$ and $60 \%-70 \%$ of $\mathrm{Mg}$ and $\mathrm{Si}$ can be released in gaseous form in C-shocks with velocities of only $\sim 45 \mathrm{~km} \mathrm{~s}^{-1}$ (May et al. 2000). It is important to stress that none of the above mentioned models has been tested with grains containing atoms of $\mathrm{Ca}$ and that these predictions are highly dependent on dust size, structure, and composition. Different results, for example, are retrieved when considering silicate or graphite grains, and if the grains are solid or porous (Jones et al. 1994; Guillet et al. 2009).

In any case, none of the present models predict total dust grain destruction in the mild shocks that occurr in the jet where gas and dust interact with matter already put into motion by the passage of previous fronts $\left(v_{\mathrm{s}} \leq 100 \mathrm{~km} \mathrm{~s}^{-1}\right.$ in the jet, Hartigan et al. 2001). This discrepancy between the predictions of shock models and the measured Ca gas-phase abundance is easily solved if we assume that the dust has been reprocessed in the disk or circumstellar region before being extracted and accelerated into jets. According to recent theoretical models and observations, the accretion disk in Herbig and Classical T Tauri Stars is populated by dust grains only ouside of the so-called dust evaporation radius, $R_{\text {evp }}$ (e.g., Isella $\&$ Natta 2005). One can thus infer that the material in the jet which is coming from a region smaller than $R_{\text {evp }}$ would not contain grains, as these have been destroyed by the stellar radiation. The dust evaporation radius is located at $\sim 0.1-1.5$ AU in Herbig stars (Eisner et al. 2007) and at $\sim 0.05-0.3$ AU in T Tauri stars (Akeson et al. 2005). Note however there are no estimates of the radius for younger and more embedded sources such as those giving rise to the $\mathrm{HH}$ jets in our sample.

On the other hand, depletion is observed at various levels along the jet, and to explain the presence of dust grains we have to assume that part of the material in the jet is coming from a region of the disk which extends beyond $R_{\text {evp }}$. Note that we are not observing ambient dust since this has been swept-out or destroyed by the passage of major bow-shocks, which have larger shock velocities (for example $v_{\mathrm{s}}$ up to $\sim 150 \mathrm{~km} \mathrm{~s}^{-1}$ in the bow of HH 1, Bally et al. 2002) and the dust reformation time-scale is much longer than the dynamical timescale of the jet, i.e. $10^{3}-10^{4} \mathrm{yr}$. Moreover, the rise in gas-phase calcium with distance from the source along the HH 111 and $\mathrm{HH} 1$ jets, and the lack of deceleration, argues against significant entrainment of ambient matter along the jet, as would be suggested by models of prompt and/or turbulent entrainment (Masson \& Chernin 1993; Stahler 1994).

Interestingly, in $\mathrm{HH} 111$, for which we could perform the analysis in the two velocity intervals, the $\mathrm{Ca}$ depletion is at a minimum in the HVI of the bow-shaped knot L (see HST images from Reipurth et al. 1997) which is also the one with the highest shock velocity $\left(v_{\mathrm{s}} \sim 60 \mathrm{~km} \mathrm{~s}^{-1}\right.$, Hartigan et al. 2001). This result suggests that the efficiency of shocks in destroying dust grains strongly depends on the shock velocity, in agreement with the predictions of theoretical models (Jones et al. 1994; Draine 2003; Guillet et al. 2009). Our estimate also indicates that this shock should be able to destroy at least $40 \%$ of the dust grains present at this position.

The proposed link between destruction efficiency and shock velocity is also supported by our finding that calcium is less depleted, or not depleted at all, along HH 83 and HH 24. These jets, in fact, show higher excitation conditions in comparison to $\mathrm{HH} 1$ and $\mathrm{HH} 111$ suggesting that their gas has undergone stronger internal shocks.

\section{Summary and conclusions}

In this paper we analyse optical spectra of a sample of HH jets located in the Orion and in the Lupus molecular clouds (HH 111, $\mathrm{HH}$ 1/2, HH 83, HH $24 \mathrm{M} / \mathrm{A} / \mathrm{E} / \mathrm{C}$, and Sz68), to investigate primarily the amount of $\mathrm{Ca}$ in the gas-phase and, in turn, the dust reprocessing in jets. Towards this aim we first apply spectral diagnostics (Bacciotti \& Eislöffel 1999) to derive the physical conditions of the gas in the jets from the line ratios of the detected forbidden lines, i.e. the values of the electron and total density, the ionisation fraction, and the temperature. These parameters are then used to estimate the expected $\mathrm{Ca}$ abundance.

We find that in the studied jets the electron density ranges between 50 and $4 \times 10^{3} \mathrm{~cm}^{-3}$. The ionisation fraction varies from a few percent in low excited knots where the [N II] emission is very faint to $40-70 \%$ in high excitation knots with strong [N II] emission. The temperature follows the same trend as the ionisation fraction, being $\sim 10^{4} \mathrm{~K}$ in the weakly ionised knots and $\sim 1.5-3 \times 10^{4} \mathrm{~K}$ in the highly ionised ones, in agreement with predictions from shock models (e.g., Hartigan et al. 1987). Finally, the total density derived by combining the retrieved values of $n_{\mathrm{e}}$ and $x_{\mathrm{e}}$ varies between $\sim 100$ and $6 \times 10^{4} \mathrm{~cm}^{-3}$.

The most interesting results are obtained for the HH 111 jet for which we derive the values of the physical parameters separately in two velocity intervals, Low $\left(v>-100 \mathrm{~km} \mathrm{~s}^{-1}\right)$ and High $\left(v<-100 \mathrm{~km} \mathrm{~s}^{-1}\right)$ (LVI and HVI). This analysis highlights many shock signatures such as $n_{\mathrm{e}}$ maxima in the HVI of the brightest knots and a sharp increase of $x_{\mathrm{e}}$ and $T_{\mathrm{e}}$ in the HVI of the strong 
knot L, which has a clear bow morphology in the HST images of Reipurth et al. (1997) and is the knot with the highest shock velocity in the jet beam (Hartigan et al. 2001).

We also analysed for the first time the faint jet powered by the young source Sz68. In this case we obtain low values for the electron and total density $\left(n_{\mathrm{e}} \sim 200-10^{3} \mathrm{~cm}^{-3}\right.$ and $\left.n_{\mathrm{H}} \sim 2-6 \times 10^{3} \mathrm{~cm}^{-3}\right)$ and intermediate values for the ionisation fraction and the temperature $\left(x_{\mathrm{e}} \sim 0.05-0.25\right.$ and $T_{\mathrm{e}} \sim 0.8 \times$ $\left.10^{4} \mathrm{~K}\right)$. For this jet we also measured proper motions of $\sim 300-600 \mathrm{~km} \mathrm{~s}^{-1}$ from which, assuming a jet radius of 100-300 AUs we derived a mass loss rate ranging between a few $10^{-8}$ and a few $10^{-7} M_{\odot} \mathrm{yr}^{-1}$, i.e. of the same order of magnitude of $\dot{M}_{\text {jet }}$ found for typical HH jets (Hartigan et al. 1994; Bacciotti \& Eislöffel 1999; Podio et al. 2006).

Using the inferred physical parameters we derive an estimate for the dust content along the jets by comparing observed and predicted ratios between calcium and sulphur lines. The derived Ca gas-phase abundance is lower than solar $\left([\mathrm{Ca}]_{\text {gas }} /[\mathrm{Ca}]_{\text {solar }} \sim\right.$ $0.2-0.85$ ) in all the jets, with the exception of $\mathrm{HH} 83$ to which, however, no dereddening has been applied, thus yielding upper limits to the $\mathrm{Ca}$ gas-phase abundance. In general, the measured $\mathrm{Ca}$ depletion is lower with respect to the interstellar medium ([Ca $]_{\text {gas }} /[\mathrm{Ca}]_{\text {solar }} \sim 0.01$ in Orion, Baldwin et al. 1991). This result suggests that mild shocks occurring along the jets are partially destroying dust grains, thus releasing calcium atoms into gaseous form. Interestingly, a higher degree of depletion is estimated in the low-excited jets (i.e. HH 111 and HH 1), while little or no depletion is found along $\mathrm{HH} 83$ and $\mathrm{HH} 24$ which show higher ionization fractions and temperatures. This seems to indicate that the efficiency of shocks in reprocessing dust grains strongly increases with shock velocity, as expected from theoretical models (e.g., Jones et al. 1994; Guillet et al. 2009). This idea is also supported by the finding that along HH 111 the depletion is minimum in the HVI of knot L, which has the largest shock velocity (Hartigan et al. 2001).

The measured values of Ca gas-phase abundance, however, are too large to be explained by dust reprocessing in shocks which are slower than $100 \mathrm{~km} \mathrm{~s}^{-1}$ (Jones et al. 1994; May et al. 2000; Guillet et al. 2009). This discrepancy between observational results and predictions of theoretical models may indicate that the material in the jet is extracted from a wide region of the disk that includes both the inner region close to the star, inside the so-called dust evaporation radius, $R_{\text {evp }}$, where the dust is destroyed by the stellar radiation (Isella \& Natta 2005), and the region beyond $R_{\text {evp }}$ from where the dust is lifted and accelerated in the flow. Modeling, however, is required to test this idea.

Acknowledgements. We are grateful to G. Pineau des Forêts, V. Guillet, and $\mathrm{S}$. Cabrit for useful discussions on dust reprocessing in shocks. We are also grateful to the referee for the attentive comments that allowed us to improve the first version of this paper. We thank the Irish Research Council for Science, Engineering and Technology which funded the work of Linda Podio. This work was partially supported by the European Community's Marie Curie Research and Training Network JETSET (Jet Simulations, Experiments and Theory) under contract MRTN-CT-2004-005592. Tom Ray was partially supported by Science Foundation Ireland through their Research Frontiers Programme.

\section{References}

Akeson, R. L., Walker, C. H., Wood, K., et al. 2005, ApJ, 622, 440 Asplund, M., Grevesse, N., \& Sauval, A. J. 2005, Cosmic Abundances as Records of Stellar Evolution and Nucleosynthesis, 336, 25 Bacciotti, F., \& Eislöffel, J. 1999, A\&A, 342, 717

Bacciotti, F., Chiuderi, C., \& Oliva, E. 1995, A\&A, 296, 185 Bacciotti, F., Mundt, R., Ray, T. P., et al. 2000, ApJ, 537, L49 Bacciotti, F., Ray, T. P., Mundt, R., Eislöffel, J., \& Solf, J. 2002, ApJ, 576, 222 Baldwin, J. A., Ferland, G. J., Martin, P. G., et al. 1991, ApJ, 374, 580 Bally, J., Heathcote, S., Reipurth, B., et al. 2002, AJ, 123, 2627 Beck-Winchatz, B., Böhm, K. H., \& Noriega-Crespo, A. 1994, PASP, 106, 1271 Beck-Winchatz, B., Böhm, K.-H., \& Noriega-Crespo, A. 1996, AJ, 111, 346 Berrington, K. A., \& Burke, P. G. 1981, Planet. Space Sci., 29, 377 Böhm, K.-H., \& Matt, S. 2001, PASP, 113, 158

Böhm, K.-H., \& Solf, J. 1985, ApJ, 294, 533

Coffey, D., Bacciotti, F., \& Podio, L. 2008, ApJ, 689, 1112

Davis, C. J., Hodapp, K. W., \& Desroches, L. 2001, A\&A, 377, 285

Draine, B. T. 1989, in Infrared Spectroscopy in Astronomy, ESA SP-290, 93 Draine, B. T. 2003 [arXiv: 0304488]

Eislöffel, J., \& Mundt, R. 1997, AJ, 114, 280

Eislöffel, J., Mundt, R., \& Böhm, K. 1994, AJ, 108, 1042

Eisner, J. A., Chiang, E. I., Lane, B. F., \& Akeson, R. L. 2007, ApJ, 657, 347

Esteban, C., Peimbert, M., García-Rojas, J., et al. 2004, MNRAS, 355, 229

Garcia Lopez, R., Nisini, B., Giannini, T., et al. 2008, A\&A, 487, 1019

Garcia Lopez, R., Nisini, B., Eislöffel, J., et al. 2009, A\&A, submitted

Guillet, V., Jones, A. P., \& Pineau Des Forêts, G. 2009, A\&A, 497, 145

Hartigan, P., \& Morse, J. 2007, ApJ, 660, 426

Hartigan, P., Raymond, J., \& Hartmann, L. 1987, ApJ, 316, 323

Hartigan, P., Morse, J. A., \& Raymond, J. 1994, ApJ, 436, 125

Hartigan, P., Morse, J. A., Reipurth, B., Heathcote, S., \& Bally, J. 2001, ApJ, 559, L157

Hartigan, P., Edwards, S., \& Pierson, R. 2004, ApJ, 609, 261

Heyer, M. H., \& Graham, J. A. 1989, PASP, 101, 816

Hudson, C. E., \& Bell, K. L. 2005, A\&A, 430, 725

Isella, A., \& Natta, A. 2005, A\&A, 438, 899

Jones, A. P. 2000, J. Geophys. Res., 105, 10257

Jones, A. P., Tielens, A. G. G. M., Hollenbach, D. J., \& McKee, C. F. 1994, ApJ, 433, 797

Keenan, F. P., Aller, L. H., Bell, K. L., et al. 1996, MNRAS, 281, 1073

Lavalley-Fouquet, C., Cabrit, S., \& Dougados, C. 2000, A\&A, 356, L41

Masson, C. R., \& Chernin, L. M. 1993, ApJ, 414, 230

May, P. W., Pineau des Forêts, G., Flower, D. R., et al. 2000, MNRAS, 318, 809 Mendoza, C. 1983, Planetary Nebulae, 103, 143

Morse, J. A., Heathcote, S., Hartigan, P., \& Cecil, G. 1993a, AJ, 106, 1139

Morse, J. A., Heathcote, S., Cecil, G., Hartigan, P., \& Raymond, J. C. 1993b, ApJ, 410, 764

Mouri, H., \& Taniguchi, Y. 2000, ApJ, 534, L63

Nisini, B., Caratti o Garatti, A., Giannini, T., \& Lorenzetti, D. 2002, A\&A, 393, 1035

Nisini, B., Bacciotti, F., Giannini, T., et al. 2005, A\&A, 441, 159

Nisini, B., Codella, C., Giannini, T., et al. 2007, A\&A, 462, 163

Podio, L., Bacciotti, F., Nisini, B., et al. 2006, A\&A, 456, 189

Raga, A. C., \& Böhm, K. H. 1986, ApJ, 308, 829

Reipurth, B., Hartigan, P., Heathcote, S., Morse, J. A., \& Bally, J. 1997, AJ, 114, 757

Rieke, G. H., \& Lebofsky, M. J. 1985, ApJ, 288, 618

Rolph, C. D., Scarrott, S. M., \& Wolstencroft, R. D. 1990, MNRAS, 242, 109

Savage, B. D., \& Sembach, K. R. 1996, ARA\&A, 34, 279

Solf, J. 1987, A\&A, 184, 322

Solf, J., \& Böhm, K. H. 1991, ApJ, 375, 618

Solf, J., Böhm, K. H., \& Raga, A. 1988, ApJ, 334, 229

Stahler, S. W. 1994, ApJ, 422, 616

van Dishoek, E. F., Blake, G. A., Draine, B. T., \& Lunine, J. I. 1993, Protostars and Planets III, 163

Zealey, W. J., Williams, P. M., \& Zinnecker, H. 1989, PASA, 8, 62 


\section{Appendix A: Physical structure of previously analysed jets}

In this appendix we present the results obtained by applying spectral diagnostics to $\mathrm{HH} 1$ (jet and bow), $\mathrm{HH} \mathrm{2,} \mathrm{HH} \mathrm{83,} \mathrm{and}$ $\mathrm{HH} 24$. These jets have been already analysed in previous papers with the same diagnostics, but are re-analysed here with higher spatial sampling $\left(\Delta x \sim 1.2^{\prime \prime}\right)$ and an improved version of the diagnostic code as explained in Sect. 3. The values presented are used for investigating dust reprocessing in these jets (see Sect. 5).

\section{A.1. $H H$ 1/2: the jet and its terminal bows}

The physical structure of the HH 1 jets and its terminal bows has been previously analysed by Solf \& Böhm (1991), Nisini et al. (2005), and Böhm \& Solf (1985), Solf et al. (1988), Eislöffel et al. (1994), Bally et al. (2002). In our observations the slit is aligned along the jet and it covers the fainter western/eastern parts of $\mathrm{HH} 1$ and $\mathrm{HH} 2$ respectively, i.e. the knots $\mathrm{B}$ and $\mathrm{G}$, which are located to the west of the HH 1 bow apex (knot F), and the knots L, J, G, B, T, and Q located to the east of the bright $\mathrm{H}$ and $\mathrm{A}$ knots in $\mathrm{HH} 2$.

The results obtained by applying the $\mathrm{BE}$ technique to the $\mathrm{HH} 1$ jet are shown in Fig. A.1. The values for the physical parameters are in a good agreement with the ones obtained in Nisini et al. (2005) but here the spatial sampling is two times better.

We could not apply the diagnostic to the terminal bows. The $\mathrm{BE}$ technique, in fact, relies on the assumption of low excitation conditions, i.e. $\mathrm{S}$ being totally ionised but there is no $\mathrm{S}^{++}$, and oxygen and nitrogen are ionised at most once (Bacciotti \& Eislöffel 1999). This assumption is satisfied for the jet where gas interacts with material already in motion from previous outflow events, but may not be correct when dealing with the terminal bows. This picture is confirmed by proper motions studies by Bally et al. (2002) which indicate shock velocities lower than $30 \mathrm{~km} \mathrm{~s}^{-1}$ in the jet and velocity jumps of up to $100-200 \mathrm{~km} \mathrm{~s}^{-1}$ in the bows. Moreover, Böhm \& Solf (1985) and Solf et al. (1988) detected many high excitation lines in HH 1 and HH 2 such as $\mathrm{O}^{++}, \mathrm{S}^{++}$, and $\mathrm{Ar}^{+++}$.

Even if in our case the slit covers the lateral part of the bows the excitation conditions may still be too high. This is indicated by the detection of the high excitation [Ar III] $\lambda 7135.8$ line in our spectra and by the fact that the [N II] lines, which are faint in the jet, show strong emission in the bows, comparable to the [S II] lines (see the upper panel of Fig. A.2).

In order to compare the physical conditions in the jet and in the bows we computed the electron density, $n_{\mathrm{e}}$, and the $[\mathrm{N} I I] /[\mathrm{OI}]$ ratio along all the slit length. The electron density does not show large differences. It varies between $0.05-4 \times 10^{3} \mathrm{~cm}^{-3}$ along all the jet. While in the jet $n_{\mathrm{e}}$ is decreasing with distance from the source, both in the HH 1 and in HH 2 bows, on the contrary, there is a decreasing trend going from the shock apex toward the source. This is expected behind a shock front and confirms the results found by Böhm $\&$ Solf (1985) and Solf et al. (1988). Our values of $n_{\mathrm{e}}$ are lower than those inferred by Böhm \& Solf (1985), however, because of the different slit alignment (on the brightest spots at the apex of HH 1 and HH 2 for Böhm \& Solf 1985, and on the bows wings in our case) and show that the density in the two bows is maximum at the apex and fades towards the wings. The $[\mathrm{N} \mathrm{II}] /[\mathrm{OI}]$ ratio is a good indicator of excitation conditions and, in particular, of the ionisation state of the gas. Figure A.2 shows that $[\mathrm{N} \mathrm{II}] /[\mathrm{O} \mathrm{I}]$ is

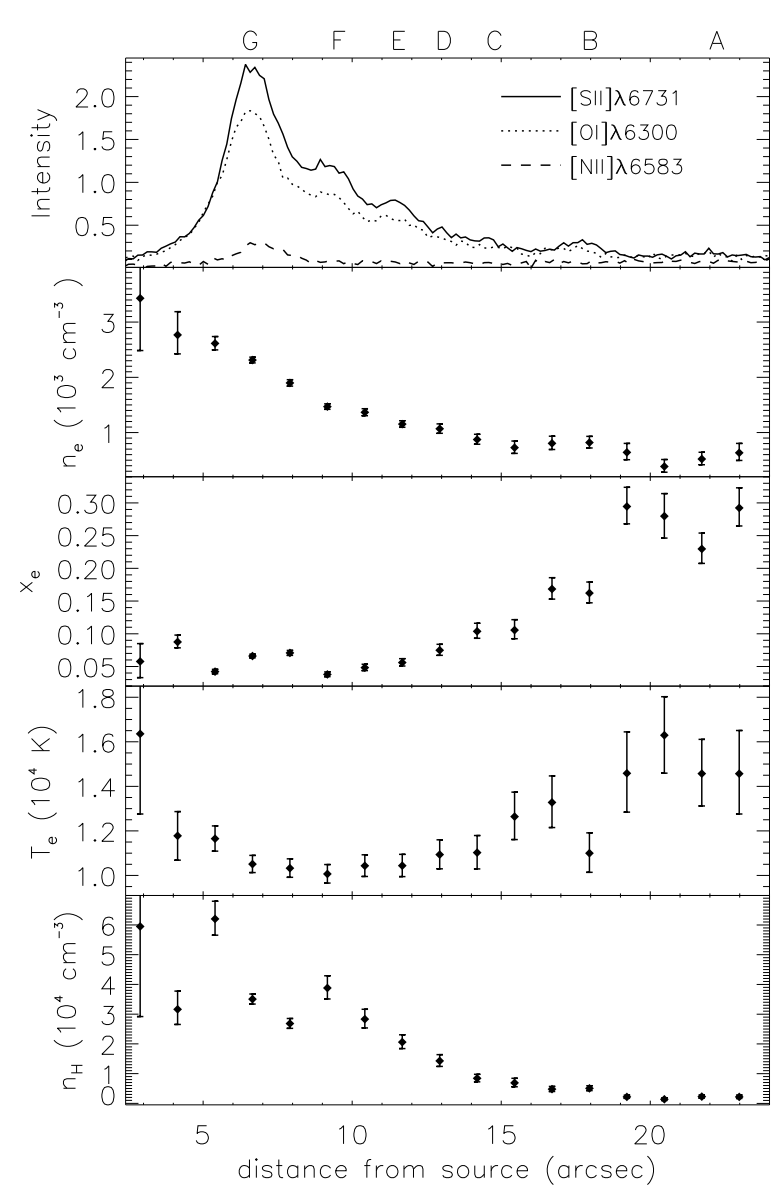

Fig. A.1. Variation of the physical parameters for the HH 1 jet as a function of distance from the source. From top to bottom panel: intensity profiles of the optical lines, the electron density, $n_{\mathrm{e}}$, in units of $10^{3} \mathrm{~cm}^{-3}$, the ionisation fraction, $x_{\mathrm{e}}$, the temperature, $T_{\mathrm{e}}$, in units of $10^{4} \mathrm{~K}$, and the total density, $n_{\mathrm{H}}$, in units of $10^{4} \mathrm{~cm}^{-3}$.

$<1$ in the jet and in knot $\mathrm{L}$, while it is $>1$ in the $\mathrm{HH} 1$ and $\mathrm{HH} 2$ bows, indicating that the excitation level is much higher in the bows, and a higher value of $x_{\mathrm{e}}$ is expected.

\section{A.2. HH 83: the physical structure of the inner knots}

The physical structure of the HH 83 jet has been already derived in Podio et al. (2006). Thanks to the high $\mathrm{S} / \mathrm{N}$ of these data, however, we obtained a sampling which is four times larger in comparison to previous results. Moreover the good quality of the data, which allowed us to properly subtract the continuum emission from the reflection nebula Re 17 (Rolph et al. 1990), and the use of the improved diagnostic code allowed us to estimate the gas physical conditions in the inner part of the jet, where emission from the $[\mathrm{N} \mathrm{II}]$ lines is comparable to the [S II] and $[\mathrm{OI}]$ emission.

The derived parameters, shown in Fig. A.3, indicate that the excitation conditions are very high in the knots close to the source (knots from A to D) with $n_{\mathrm{e}} \sim 400-700 \mathrm{~cm}^{-3}$, and high values of the ionisation fraction and temperature $\left(x_{\mathrm{e}} \sim 0.4-0.7\right.$, and $T_{\mathrm{e}} \sim 1.5-2 \times 10^{4} \mathrm{~K}$ ). 


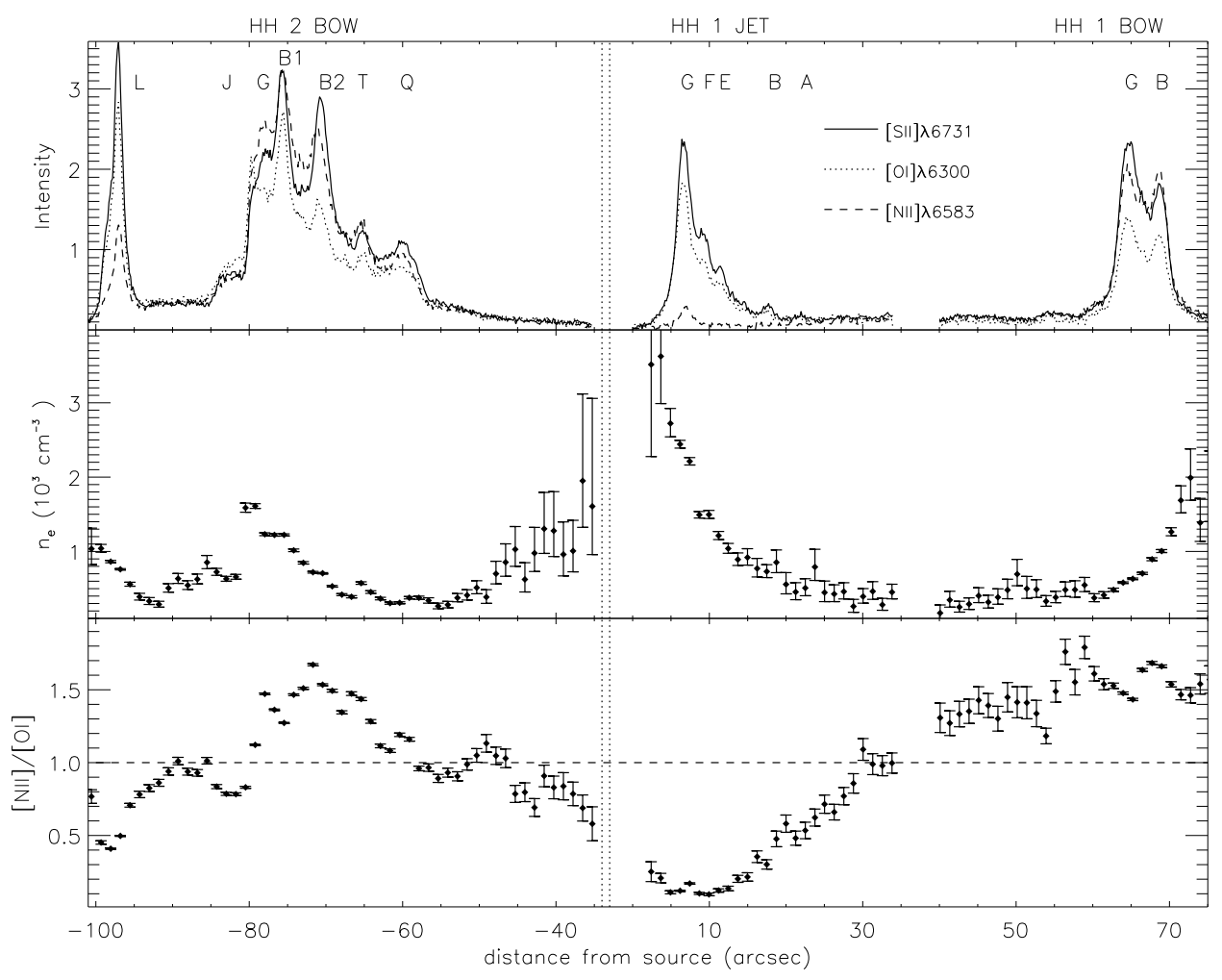

Fig. A.2. Analysis of the excitation conditions in the HH 1 jet and its terminal bows HH 1 and HH 2 as a function of distance from the source. From top to bottom panel: intensity profiles of the optical lines, the electron density, $n_{\mathrm{e}}$, in units of $10^{3} \mathrm{~cm}^{-3}$, and the [N II $] /[\mathrm{OI}] \mathrm{ratio}$.

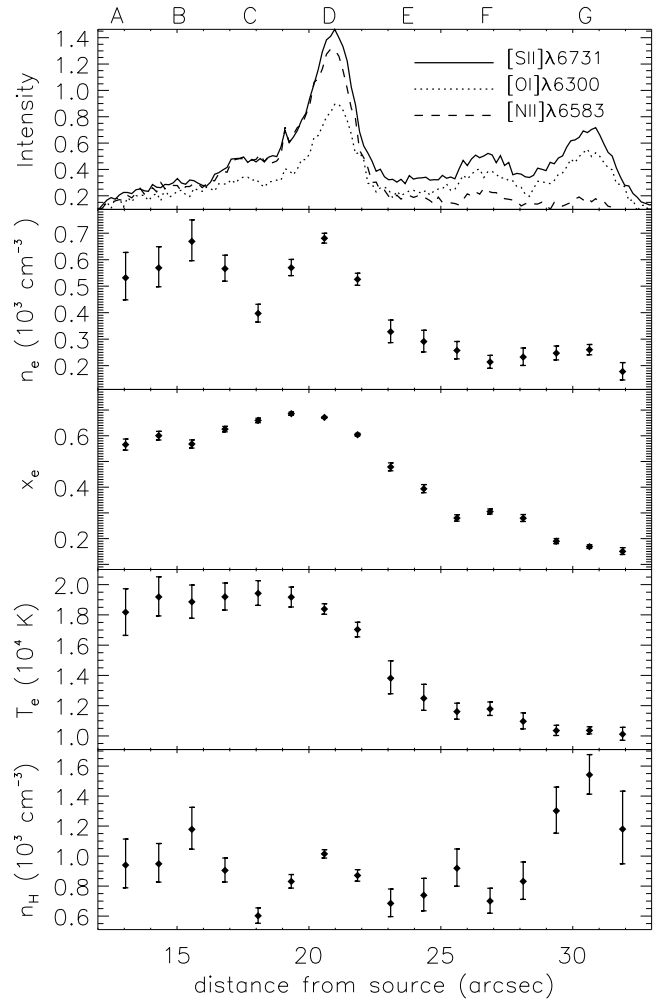

Fig. A.3. Variation of the physical parameters for the HH 83 jet as a function of distance from the source. From top to bottom panel: intensity profiles of the optical lines, the electron density, $n_{\mathrm{e}}$, in units of $10^{3} \mathrm{~cm}^{-3}$, the ionisation fraction, $x_{\mathrm{e}}$, the temperature, $T_{\mathrm{e}}$, in units of $10^{4} \mathrm{~K}$, and the total density, $n_{\mathrm{H}}$, in units of $10^{3} \mathrm{~cm}^{-3}$.

\section{A.3. The HH 24 jets}

The physical parameters along the jets HH 24 C, E, and A, have already been derived in Bacciotti \& Eislöffel (1999) and Podio et al. (2006). In our observations the slit has been aligned along the axis HH 24 M-A-E and thus only partially covers the knots of the HH 24 C jet up to 40" (knot C6). This is why the line profiles in the upper panel of Fig. A.4 show fainter emission in the knots of group C, contrary to what was found in Podio et al. (2006), where the slit was aligned along the $\mathrm{HH} 24 \mathrm{C}$ jet. The variation of the physical parameters obtained by applying the BE technique is shown in Fig. A.4. The sampling is improved by around one third with respect to previous analyses (Bacciotti \& Eislöffel 1999; Podio et al. 2006) allowing us to highlight the different excitation conditions in the various groups of knots detected in the HH 24 complex (HH 24 A, HH 24 M/E, and HH 24 C) and supporting the idea that these knots may belong to different jets. 
L. Podio et al.: Physical structure and dust reprocessing in a sample of $\mathrm{HH}$ jets, Online Material $p 3$

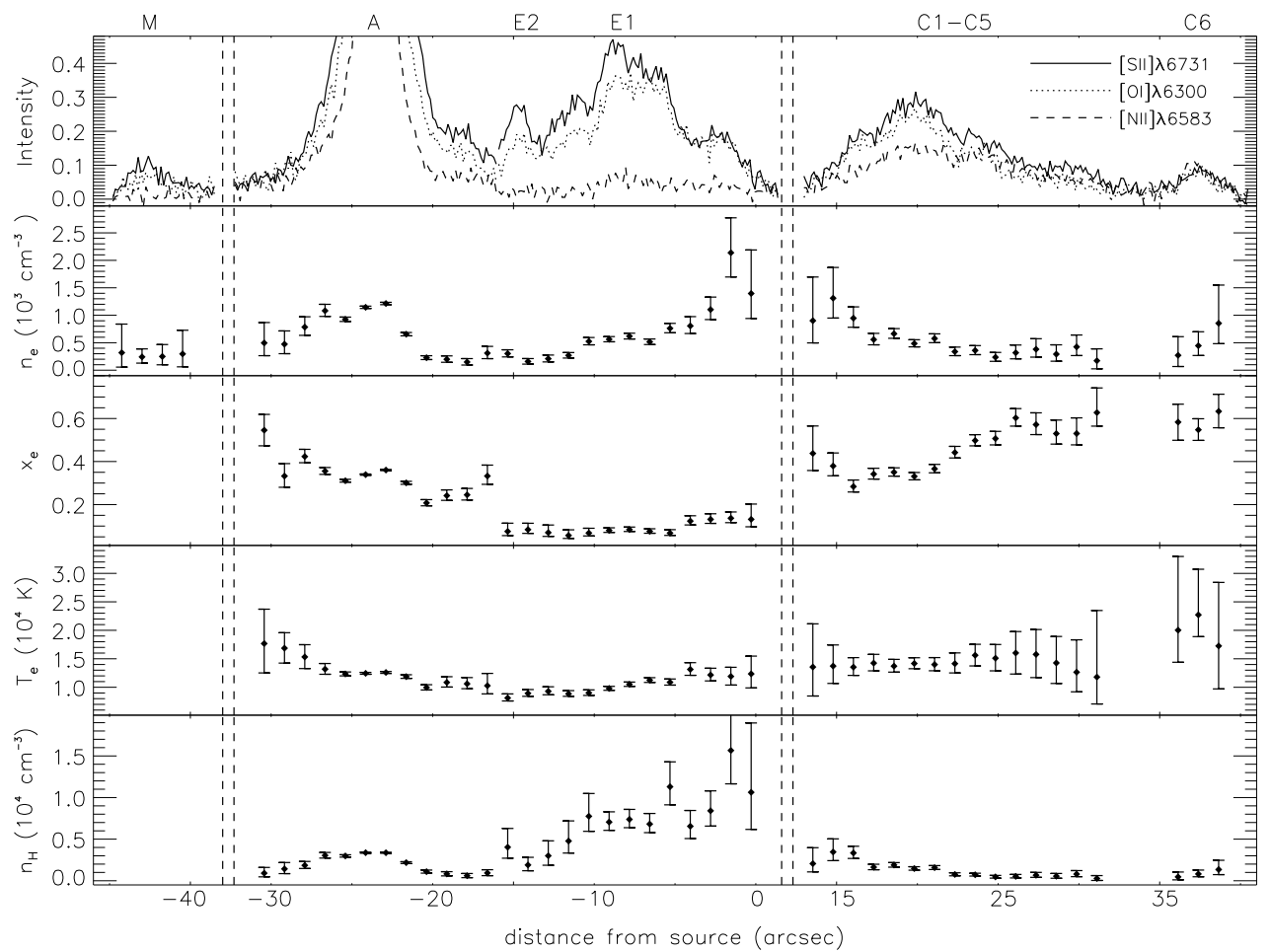

Fig. A.4. Variation of the physical parameters for the $\mathrm{HH} 24 \mathrm{C} / \mathrm{E}$ jet as a function of distance from the source. From top to bottom panel: intensity profiles of the optical lines, the electron density, $n_{\mathrm{e}}$, in units of $10^{3} \mathrm{~cm}^{-3}$, the ionisation fraction, $x_{\mathrm{e}}$, the temperature, $T_{\mathrm{e}}$, in units of $10^{4} \mathrm{~K}$, and the total density, $n_{\mathrm{H}}$, in units of $10^{4} \mathrm{~cm}^{-3}$. 